\title{
20 Jahre nach dem Mauerfall: Arbeitslosigkeit in ostdeutschen Regionen
}

\author{
Uwe Blien · thi Hong Van Phan · Klara Kaufmann · Steffen Kaimer
}

Angenommen: 21. Juli 2010 / Online veröffentlicht: 3. September 2010

(C) Institut für Arbeitsmarkt- und Berufsforschung 2010

Zusammenfassung 20 Jahre nach dem Mauerfall sind Ökonomie und Arbeitsmarkt in Deutschland immer noch von einem starken West-Ost-Gefälle geprägt. Damit ist ein großer Unterschied in der Arbeitslosigkeit verbunden, der sich allerdings in den vergangenen Jahren etwas reduziert hat. Dieser Unterschied ist das eine Thema des vorliegenden Artikels. Sein zweiter Gegenstand ist die starke Differenzierung der Arbeitsmarktlagen innerhalb Ostdeutschlands. Zum Verständnis der Situation werden neuere regionalökonomische Theorien herangezogen. Außerdem wird die Entwicklung der regionalen Arbeitslosigkeit in einem räumlichen ökonometrischen Modell analysiert.

\section{0 years after the fall of the Berlin wall: unemployment in regions of Eastern Germany}

\begin{abstract}
Themes of the paper are differences in unemployment rates between East and West Germany and the spatial differentiation in the East. 20 years after the fall of the Berlin Wall there is still a strong division between the economies and labour markets of Eastern and Western Germany. In the last few years, however, unemployment has been reduced especially in the East. Within Eastern Germany a strong variation of labour market situations is visible, which has been relatively stable in time. In the paper special approaches of regional economics are used to understand the situation. In addition, a spatial econometrics framework is applied.
\end{abstract}

U. Blien ( ) t t. H. V. Phan · K. Kaufmann · S. Kaimer Institut für Arbeitsmarkt- und Berufsforschung (IAB), Regensburger Str. 104, 90478 Nürnberg, Deutschland E-Mail: uwe.blien@iab.de

\section{Einführung}

20 Jahre nach dem Mauerfall bleiben die ostdeutschen Regionen immer noch in auffälliger Weise hinter den westdeutschen zurück: Das Niveau der Einkommen aus sozialversicherungspflichtiger Beschäftigung ist im Jahre 2008 um $22 \%$ niedriger als im Westen (Quelle: eigene Berechnungen mit Daten der IAB-Beschäftigtenstichprobe). Noch stärker fällt auf, dass die Arbeitslosigkeit nun schon über lange Zeit hinweg doppelt so hoch ist wie im Westen. Personen, die in ostdeutschen Regionen leben, sind folglich in mindestens zweifacher Weise benachteiligt: Zum einen finden sie viel schwerer einen Arbeitsplatz, weil die Arbeitslosigkeit viel höher ist, zum anderen ist ihr Einkommen niedriger.

Wie können derart große Unterschiede innerhalb eines Landes über einen so langen Zeitraum bestehen bleiben? Zwanzig Jahre sind eine lange Zeit. Die neoklassischen Standardansätze der ökonomischen Theorie ließen erwarten, dass sich Arbeitsmarktdisparitäten ausgleichen würden, selbst ohne unterstützende wirtschaftspolitische Maßnahmen. Stattdessen beobachtet man eine große Stabilität in den Unterschieden. Diese betreffen auch die kleinräumige Ebene. Ostdeutschland ist kein homogener Raum, es gibt vielmehr Regionen, die den Anschluss an den Westen durchaus schaffen, andere hängen jedoch weit zurück (vgl. Abb. 1).

Aus diesen Gründen wollen wir uns im Folgenden zunächst mit dem globalen Arbeitslosigkeitsgefälle zwischen Ost- und Westdeutschland befassen. Im zweiten Teil unseres Beitrags sollen dann die internen regionalen Differenzierungen in Ostdeutschland näher beleuchtet werden. Die gemeinsame Fragestellung betrifft also die regionale Arbeitslosigkeit in Ostdeutschland einerseits im Verhältnis zum Westen, andererseits in der internen Differenzierung. 
Abb. 1 Arbeitslosenquote November 2009

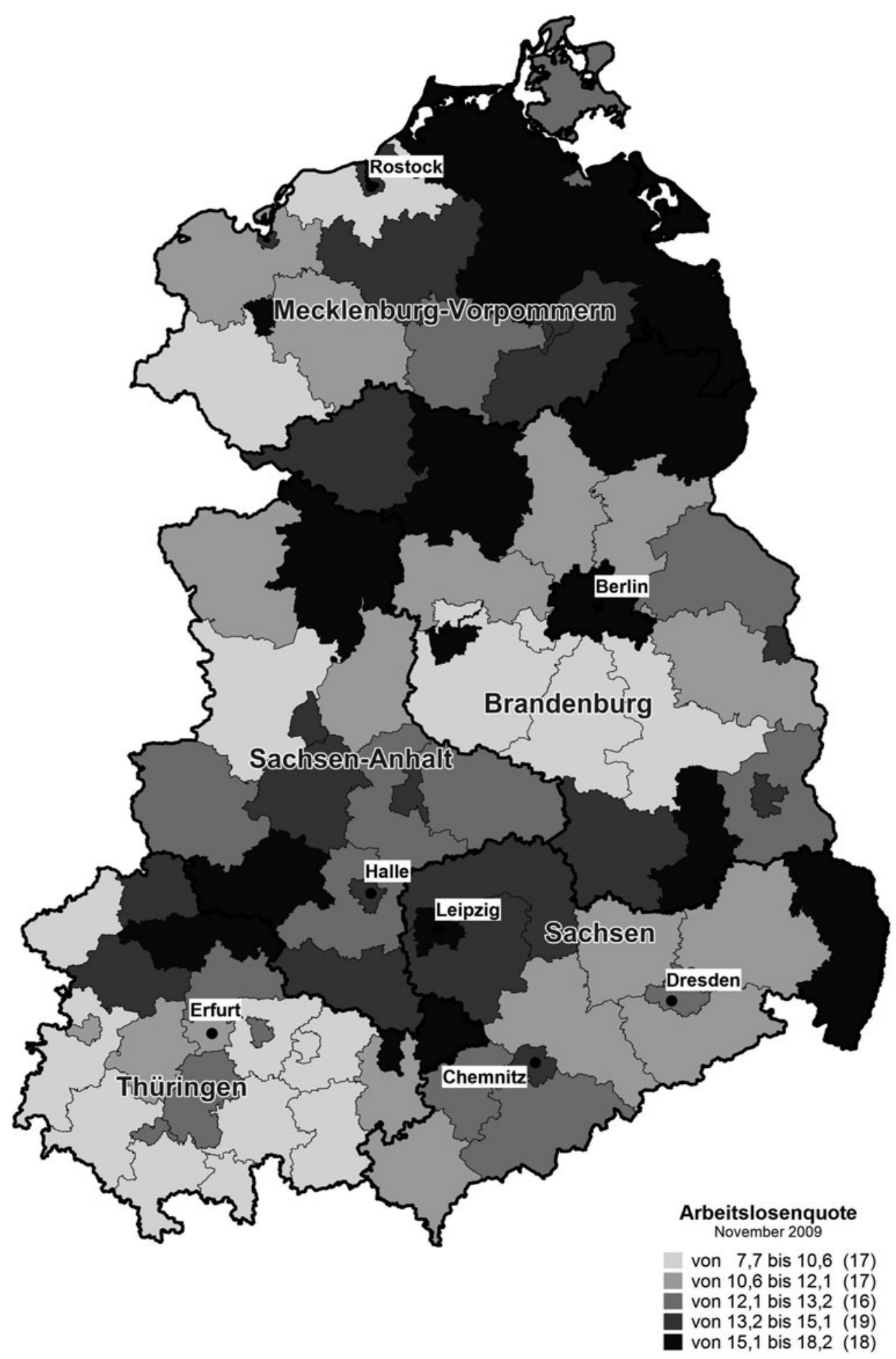

Zum Verständnis der Situation werden neuere regionalökonomische Theorien herangezogen. Außerdem wird die Entwicklung der regionalen Arbeitslosigkeit innerhalb Ostdeutschlands in einem räumlichen ökonometrischen Modell analysiert.

\section{Das Gefälle von West nach Ost}

Trotz der schon angesprochenen Tendenz zur Beharrung bei vielen wichtigen Arbeitsmarktindikatoren ist festzuhalten, dass Ostdeutschland in bestimmten wichtigen Bereichen 
gegenüber dem Westen aufgeholt hat, zumeist jedoch ohne das Niveau der alten Länder voll zu erreichen. Für die Zeit unmittelbar nach der deutschen Vereinigung gibt es keine Information aus der Beschäftigtenstatistik. Verwendet man stattdessen das verfügbare Einkommen der amtlichen Statistik pro Einwohner als Indikator, so hat sich der Abstand der neuen Länder (ohne Berlin) von 42,9 \% (1991) inzwischen auf 18,3\% (2008) reduziert (BMWi 2009). Die Menschen in Ostdeutschland profitieren zudem davon, dass seit der Vereinigung viele öffentliche Leistungen verbessert wurden. Die Städte und Dörfer wurden saniert und die Infrastruktur ausgebaut.

Ein wichtiger Indikator, bei dem über längere Zeit hinweg kein solcher Aufholprozess festzustellen ist, betrifft das Einkommen, das seit 1996 stagniert, wenn man es am Maßstab Westdeutschland misst. Die (neo-),,klassischen“ Ansätze der Wachstumstheorie ließen erwarten, dass sich derartige Unterschiede über Konvergenzprozesse angleichen würden. Aus empirischen Untersuchungen leiteten Barro u. Sala-i-Martin (1991) ab, dass Unterschiede im Wachstumsprozess zum Ausgleich der Produktivitäten führen, dass diese Angleichung jedoch langsam abläuft. Sie sollte den Unterschied um rund $2 \%$ pro Jahr reduzieren, wie schon von Barro (1991) für den Fall der neuen Länder geltend gemacht wurde. Allerdings beziehen sich die verwendeten Untersuchungen auf den Fall der sogenannten „ $\beta$-Konvergenz“. Dieses Konzept wurde alsbald als nicht hinreichend trennscharf kritisiert (siehe Kasten 1), sodass die für andere Länder gefundenen und auf das vereinigte Deutschland übertragenen empirischen Belege nicht als konklusiv gelten können.

Kasten 1

\section{Ansätze zur Messung der Konvergenz von Regionen}

Barro u. Sala-i-Martin (1991) führten Untersuchungen zur Konvergenz des Volkseinkommens zwischen Nationen durch, indem sie in eine Regressionsschätzung für die Wachstumsrate des Volkseinkommens als abhängiger Variablen das Niveau des Einkommens zum Beginn des betrachteten Zeitraums als wichtigste unabhängige Variable aufnahmen. Die Schätzgleichung hatte demnach folgende Gestalt (die Punkte deuten weitere kontrollierende Variablen an, die gegebenenfalls zusätzlich aufgenommen wurden):

$$
\frac{y_{t o+1}-y_{t o}}{y_{t o}}=\beta_{0}+\beta_{1} y_{t o}+\ldots+\varepsilon .
$$

Für den Koeffizienten $\beta_{1}$ in dieser Gleichung wurde ein negativer Wert gefunden. Dies wurde so interpretiert, dass Nationen (bzw. in der weiteren Übertragung: Regionen) mit einem hohen Einkommen relativ langsamer und Nationen mit niedrigem Einkommen relativ schneller wachsen als der Durchschnitt. In der Folge wurde an diesem Befund, der eben $\beta$-Konvergenz getauft wurde, von verschiedener Seite Kritik geübt (vgl. z. B. Friedman 1992; Quah 1993; Bliss 1999). Tatsächlich kann formal gezeigt werden, dass ein negativer Koeffizient $\beta_{1}$ in Gl. $1^{*}$ nur eine notwendige, aber keine hinreichende Bedingung dafür ist, dass sich die Regionen aufeinander zu entwickeln, d.h. die Streuung einer Population abnimmt. Man ersieht aus der Gleichung, wie es möglich ist, dass die Varianz einer Population (z. B. von Regionen) selbst bei negativem $\beta_{1}$ im Zeitablauf steigen kann: In der Regressionsgleichung ist ein Fehlerterm $\epsilon$ enthalten, der die Streuung der abhängigen Variablen ebenfalls beeinflusst.

Das für den Fall der Konvergenz konklusive Konzept besteht vielmehr im Vergleich der Standardabweichung des Volkseinkommens für verschiedene Zeitpunkte, wie später auch von Barro u. Sala-i-Martin (2004) zugestanden wurde. Trotzdem sieht man immer noch häufig Anwendungen des Konzepts der $\beta$-Konvergenz, ohne dass die damit verbundenen Beschränkungen reflektiert würden. Kann eine Verringerung der Standardabweichung festgestellt werden, so wird dies , $\sigma$-Konvergenz“ genannt. Im vorliegenden Kontext ist nicht das Einkommen die interessierende Variable, sondern die Arbeitslosigkeit, für die das methodische Argument in analoger Weise gilt.

Die Arbeitslosigkeit ist ein weiterer wichtiger Indikator, der große und stabile Unterschiede zwischen Ost und West anzeigt. Grundsätzlich setzen niedrige Werte bei der Arbeitslosigkeit voraus, dass sich Beschäftigung für die Kapitaleigner lohnt und im Hinblick auf Kosten und Güternachfrage entsprechend günstige Bedingungen herrschen. Abbildung 2 stellt die zeitliche Entwicklung der Arbeitslosenquote (Juni-Werte) für die beiden Landesteile dar. Die Abbildung macht deutlich, dass die Arbeitslosigkeit in Ostdeutschland von einem strukturellen Problem betroffen ist: Für den größten Teil des betrachteten Zeitraums blieb sie auf einem sehr hohen Niveau jenseits konjunktureller Einflüsse. Ein hoher Sockel bestand auch in Zeiten der Hochkonjunktur fort: Der „New-Economy-Boom“ hatte eine Phase konjunktureller Erholung auf dem Arbeitmarkt zur Konsequenz, die bis ungefähr 2001 andauerte. In dieser Zeit nahm die Arbeitslosigkeit im Westen deutlich stärker ab als im Osten.

Die jüngste konjunkturelle Erholung nach 2005 erbrachte eine Bestätigung der „Faustregel“, dass die Arbeitslosigkeit im Osten ungefähr doppelt so hoch ist wie im Westen (vgl. Bogai u. Hirschenauer 2008), da die Arbeitslosigkeit in Ostdeutschland ungefähr proportional sank. Da sich dadurch 
Abb. 2 Arbeitslosenquote nach Jahren

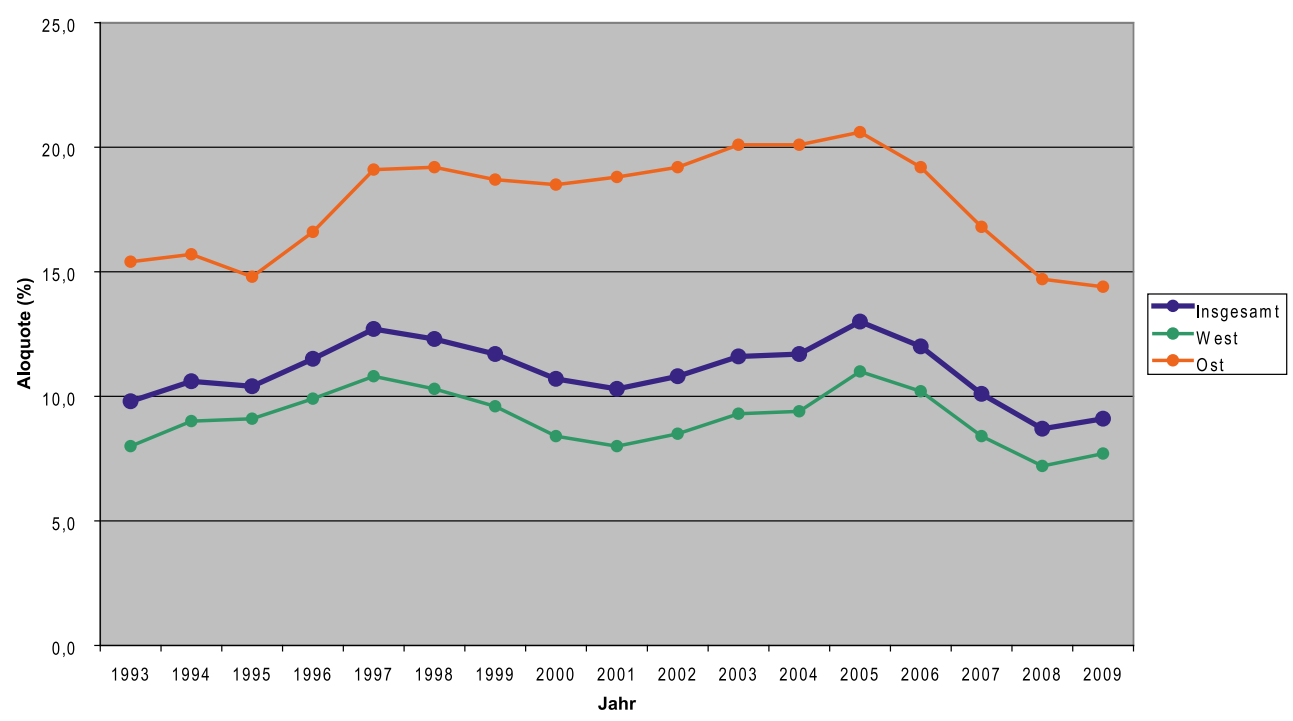

die absoluten Unterschiede zwischen den beiden Landesteilen reduzieren, implizierte dies einen wesentlich steileren Entwicklungspfad als im Westen, wie Abb. 2 zeigt. Die Tendenz zum Abbau wird nun am Ende des Beobachtungszeitraums durch die Krise der Weltwirtschaft deutlich verlangsamt; immerhin fällt die Arbeitslosenquote in Ostdeutschland selbst in einer Zeit, in der die westdeutschen Regionen deutlich steigende Werte aufweisen.

Warum setzten sich Tendenzen zur Konvergenz der beiden Landesteile nicht schon eher und wesentlich stärker durch? Nach den Standardansätzen der ökonomischen Theorie hätte man erwarten können, dass Ostdeutschland leichter Kapital anlocken können würde als Westdeutschland. Die niedrigeren Löhne in Ostdeutschland bedeuteten einen Kostenvorteil für Investoren. Nachteile bei der Infrastrukturausstattung wurden zunehmend durch öffentliche Investitionen ausgeglichen. Als Folge müssten sich Löhne und Beschäftigung dem Niveau in Westdeutschland angleichen. Der Ausgleich der Arbeitslosenquoten müsste sogar noch schneller erfolgen: Arbeitslose aus Ostdeutschland sollten zunehmend im Westen Arbeit finden und auf diese Weise den Bestand der Arbeitskräfte in Ostdeutschland verkleinern. Als Folge dieser Mobilität sollte sich die Arbeitslosenquote zwischen den Landesteilen angleichen.

Stattdessen beobachtet man ein merkwürdige Entwicklung: Obwohl so viele Menschen aus Ostdeutschland abwandern, dass einzelne Regionen sich zunehmend entleeren, fällt die Arbeitslosigkeit kaum! Zwischen 1990 und 2007 verringerte sich vor allem durch Abwanderung die Bevölkerung in Ostdeutschland mit Berlin um nicht weniger als 8,2\%, dies sind 1,5 Mio. Personen (Röhl 2009; Heiland 2004). Aus regionalökonomischer Sicht gibt es jedoch Erklärungen für dieses scheinbare Paradoxon. Eine solche besteht darin, dass Abwanderung gegensätzliche Effekte hat. Sie verkleinert den Bestand an Arbeitskräften in Ostdeutschland und senkt durch die Reduzierung des Arbeitskräfteangebots direkt die Arbeitslosenquote. Darüber hinaus gibt es jedoch einen indirekten Effekt, weil Abwanderung auch die kaufkräftige Nachfrage in den ostdeutschen Regionen schwächt. Das Marktpotenzial der ostdeutschen Regionen wird reduziert. Mit zunehmender Abwanderung verkleinert sich für Firmen der Anreiz, in Ostdeutschland zu produzieren, zumindest dann, wenn sie auch auf den lokalen Markt ausgerichtet sind. Stattdessen können viele Güter im Westen produziert und dann über die inzwischen hervorragenden Verkehrsverbindungen nach Osten geschafft werden.

Abwanderung hat demnach zwei Wirkungen, eine direkte, die die Arbeitslosigkeit senkt, und eine indirekte, die das Marktpotenzial der Regionen schwächt und dadurch die Arbeitslosigkeit über einen Umweg erhöht. Welcher der beiden Effekte stärker ausfällt, kann abstrakt nicht vorhergesagt werden. Im Falle von Ostdeutschland scheinen sich die beiden Wirkungen jedoch nahezu die Waage zu halten. Die Folge ist, dass die Arbeitslosigkeit hoch bleibt.

Zur Problemanalyse wird häufig Krugmans New Economic Geography herangezogen (Krugman 1991), in der es stabile Gleichgewichte zwischen einem Zentrum und einer Peripherie geben kann. Die Theorie baut auf monopolistischer Konkurrenz, wachsenden Skalenerträgen und mobilen Produktionsfaktoren auf. Sie kennt zwei Arten von Externalitäten: Arbeitskräfte gehen dorthin, wo die Produktion stattfindet und die Produktion zieht zur kaufkräftigen Nachfrage, d.h. dorthin, wo es viele Arbeitskräfte gibt. Agglomerative Tendenzen basieren in dieser Theorie auf monetären Effekten, die sich aus der endogenen Marktgröße ergeben. 
Brakman u. Garretsen (1993, 1994) argumentierten schon bald nach der deutschen Vereinigung auf der Grundlage dieser Theorie, dass Ostdeutschland im Verhältnis zum Westen die Rolle der benachteiligten Peripherie spielen könnte, die für Investoren nicht als Produktionsstandort interessant wäre. Diese würden sich vielmehr im Zentrum, d. h. im Westen, niederlassen, wo das Marktpotenzial, d. h. die kaufkräftige Nachfrage, entsprechend größer wäre. Der Ausbau der Infrastruktur, d. h. vor allem der Verkehrswege nach Osten, würde dann nicht zum Aufbau einer entsprechenden produktiven Kapazität beitragen, sondern im Gegenteil die relative Position des Westens stärken, weil man den Osten dann um so leichter mit Waren beliefern könnte, ohne dort zu produzieren.

Divergente Prozesse, die ähnlich denen der Neuen Ökonomischen Geografie wirken, beschreibt Uhlig (2006a,b) in einem Modellrahmen, der andere kausale Abläufe kennt. Einen Überblick zu weiteren neueren Ansätzen aus dem Umfeld der New Economic Geography und darüber hinaus bieten Duranton u. Puga (2004). Inzwischen wurden viele neue theoretische Ansätze entwickelt, die andere Mechanismen der Agglomeration kennen. Dabei wird häufig auf sogenannte MAR-Externalitäten (Marshall-Arrow-RomerExternalitäten) reflektiert. Dies sind Wissens-Spillovers sowie Nutzung eines gemeinsamen Arbeitskräftepools und von Vorprodukten. Es ist eine schwierige empirische Aufgabe, diese Effekte zu identifizieren (die für Westdeutschland von Dauth 2010 unternommen wird).

Die für Ostdeutschland feststellbare Abwanderung verstärkt die Rolle des Ostens als Peripherie des Westens, weil sie das Marktpotenzial der betreffenden Regionen weiter schwächt. Insbesondere Südekum (2005) hat die New Economic Geography dadurch erweitert, dass er Arbeitslosigkeit in ihr Gebäude integriert hat. Abwanderung aus einer peripheren Region hat einen direkten Effekt auf die Arbeitslosigkeit in dem betreffenden Raum, weil sie das Arbeitskräfteangebot reduziert, aber sie hat einen entgegenwirkenden indirekten Effekt, weil sie das Marktpotenzial der betroffenen Regionen reduziert.

Vorschläge für Lösungen der Arbeitsmarktprobleme Ostdeutschlands, die pauschal darin bestehen, dass Arbeitslose doch besser in prosperierende Regionen Deutschlands abwandern sollten, erscheinen daher nicht als angemessen. Sie könnten das regionale Gefälle zwischen den beiden Landesteilen weiter vergrößern. Im Zusammenhang des sogenannten ENDOR-Projekts (Blien et al. 2003) wurde im IAB eine Bestandsaufnahme und umfassende Analyse der regionalen Entwicklungsbedingungen Ostdeutschlands durchgeführt. $\mathrm{Zu}$ diesem Zeitpunkt, zu Beginn des vergangenen Jahrzehnts, wurde eine moderat günstige Entwicklungsperspektive für die ostdeutschen Regionen als wahrscheinlich angesehen, weil sich einige wesentliche $\mathrm{Pa}$ rameter zugunsten des Ostens verbessert haben und weiter verbessern. Dies gilt für Lohnstückkosten, Infrastrukturausstattung und Innovationsfähigkeit. Die Beurteilung scheint sich jetzt zu bestätigen, da die Arbeitslosigkeit niedriger ist als zu jedem Zeitpunkt seit 1993.

\section{Regionale Disparitäten innerhalb Ostdeutschlands}

Nun ist Ostdeutschland kein homogener Raum, wie ein Blick auf die Karten für Landkreise und kreisfreie Städte zu diesem Artikel zeigt. Abbildung 1 verdeutlicht die aktuelle Verteilung der Arbeitslosigkeit nach Kreisen. Entlang der ehemaligen innerdeutschen Grenze und südlich von Berlin finden sich Regionen, deren Arbeitslosigkeit auch im Westen nicht aus dem Rahmen fallen würde. Im Gegensatz dazu stehen vor allem ländliche Gebiete, weit ab von günstigen Verkehrsverbindungen zum Westen, die nach wie vor von einer tiefen Arbeitsmarktkrise betroffen sind.

Die verwendeten Daten kommen aus der Arbeitslosenstatistik der Bundesagentur für Arbeit. Für den Zeitvergleich mussten die Kreisdaten auf den aktuellen Gebietsstand umgeschätzt werden. Wir haben dazu ein Verfahren verwendet, das auf der RAS-Methode aufbaut (dies ist ein Entropieoptimierungsverfahren, vgl. Blien u. Graef 1998).

Hinter den Disparitäten bei der Arbeitslosigkeit stehen vor allem Unterschiede in der Beschäftigungsentwicklung. Abbildung 3 stellt dazu die Wachstumsrate für die sozialversicherungspflichtige Beschäftigung dar. In allen Kartendarstellungen wurde das nach den letzten Gebietsreformen geltende Raster der 87 ostdeutschen kreisfreien Städte und Landkreise zugrunde gelegt: Abb. 4 gibt den historischen Stand der Arbeitslosigkeit für den Juni 1993 wider, Abb. 5 zeigt die absolute Veränderung der Arbeitslosenquote (Differenz der Werte zwischen Juni 1993 und Juni 2009). Abbildung 6 stellt die Veränderungsrate dar (Differenz der Werte über den gleichen Zeitraum, dividiert durch das Niveau von 1993). Abbildung 7 zeigt die Entwicklung der Arbeitslosigkeit am oberen Rand der betrachteten Zeitperiode (Differenz der Werte zwischen November 2008 und November 2009).

Bei der Beschäftigungsentwicklung werden bereits enorme Unterschiede deutlich. Obwohl der eigentliche „Vereinigungsschock“ auf die Beschäftigung nicht mehr in den Daten enthalten ist, reicht 1994 bis 2008 die Spannweite von einem Einbruch um $38 \%$ im Spree-Neiße-Kreis, der die Stadt Cottbus einschließt, bis hin zu einem Zugewinn von $18 \%$ in Bad Doberan, das ist die Region um Rostock. Berlin weist übrigens einen Abbau von $16 \%$ auf. Dies liegt dicht an dem Wert für Ostdeutschland (ohne Berlin) von insgesamt $18,1 \%$. Bei der Beschäftigung wurde ein etwas kürzerer Betrachtungszeitraum als bei der Arbeitslosigkeit zugrunde gelegt, da regionale Daten für 1993 und für 2009 nicht mit der hinreichenden Genauigkeit zur Verfügung stehen. 
Nimmt man die anderen Kartendarstellungen zur Diagnose hinzu, so zeigt sich die Andeutung einer räumlichen Struktur konzentrischer Ringe mit Berlin als Zentrum. In Berlin ergibt sich eine durchschnittliche Wachstumsrate der Beschäftigung, im Umland eine eher positive. Eine Ausnahme sind jene Regionen, die nach Norden an Ostberlin anschließen. Weiter nach außen folgt ein
„Ring“ aus ländlichen Regionen mit überwiegend hohem Beschäftigungsabbau. Daran schließen sich Regionen mit heterogener Lage an. Diese Struktur wird überlagert durch ein West-Ost-Gefälle: Richtung Westdeutschland gibt es viele Regionen mit besserer Entwicklung, während an der Grenze zu Polen ungünstige Lagen dominieren. Letzteres wird noch deutlicher, wenn man das Beschäftigungs-
Abb. 3 Beschäftigungswachstum 1994-2008

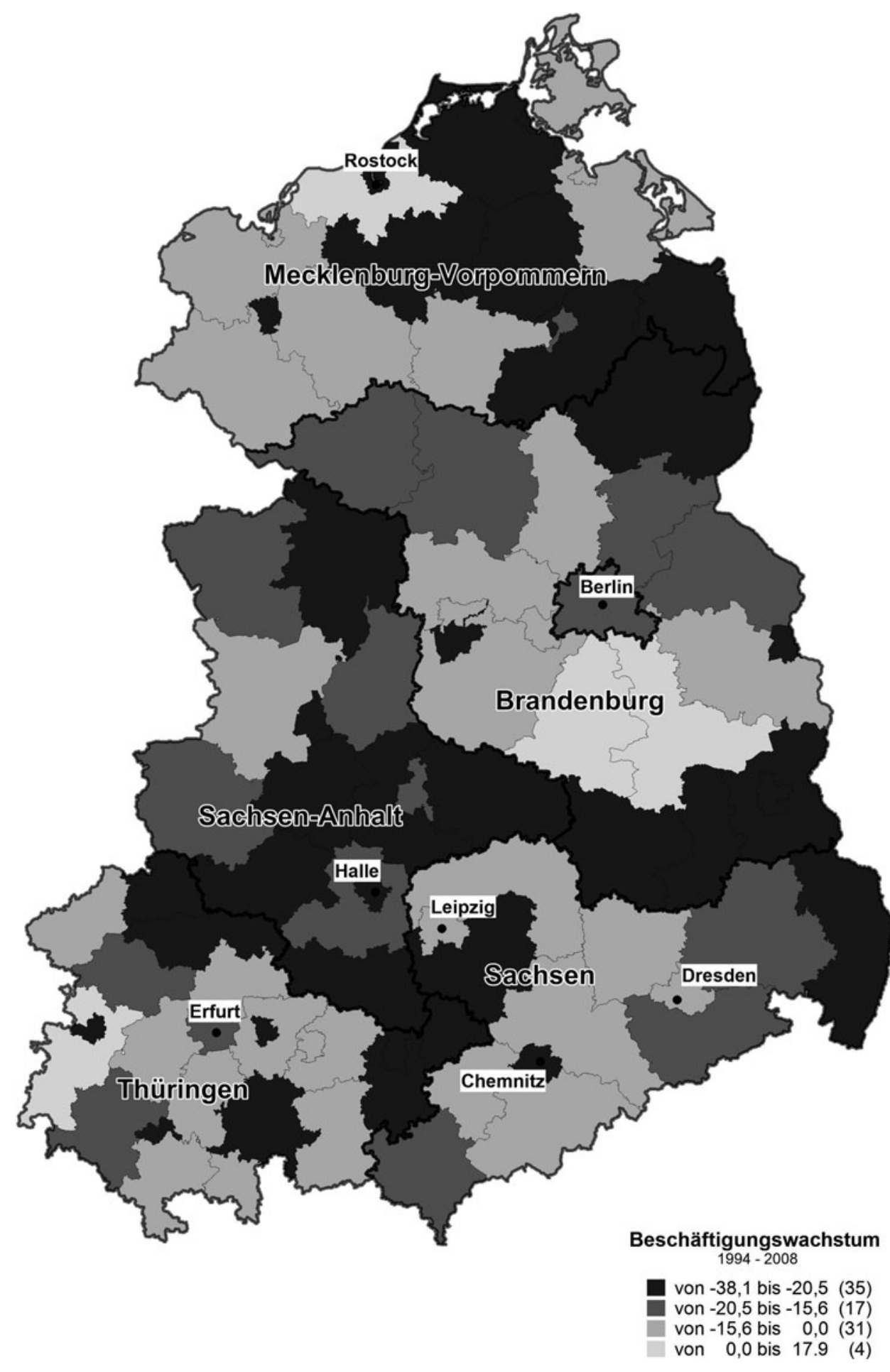


Abb. 4 Arbeitslosenquote Juni 1993

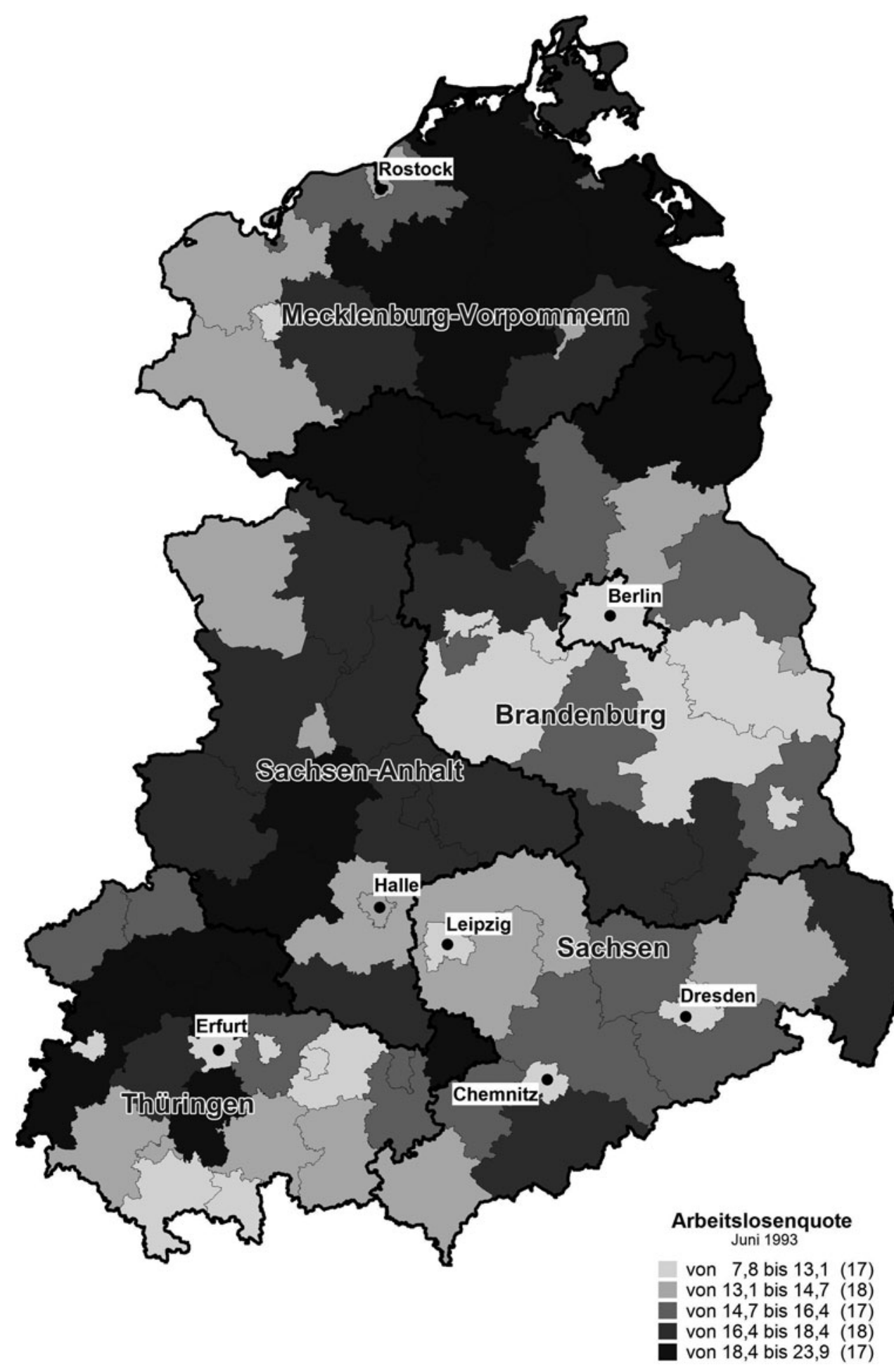

wachstum nicht auf 1994, sondern auf 1998 als Basisjahr bezieht. In diesem Fall sind nahezu alle Regionen an der Grenze von eher ungünstigen Entwicklungen betroffen. Die (Groß-)Städte in Ostdeutschland entwickeln sich heterogen: Während Leipzig und Dresden eher positiv auffallen, stellen Halle und Frankfurt eher Negativbeispiele dar.
Betrachtet man nicht die Entwicklung der Beschäftigung, sondern die Veränderung der Arbeitslosigkeit (Abb. 5), fällt generell auf, dass die Metropolen eine negative Entwicklung durchlaufen. Hier findet in bestimmtem Grade eine Angleichung zum Westen der Republik statt, wo die Großstädte überwiegend höhere Arbeitslosenquoten aufweisen als 
Abb. 5 Veränderung der Arbeitslosenquote Juni 1993 bis Juni 2009

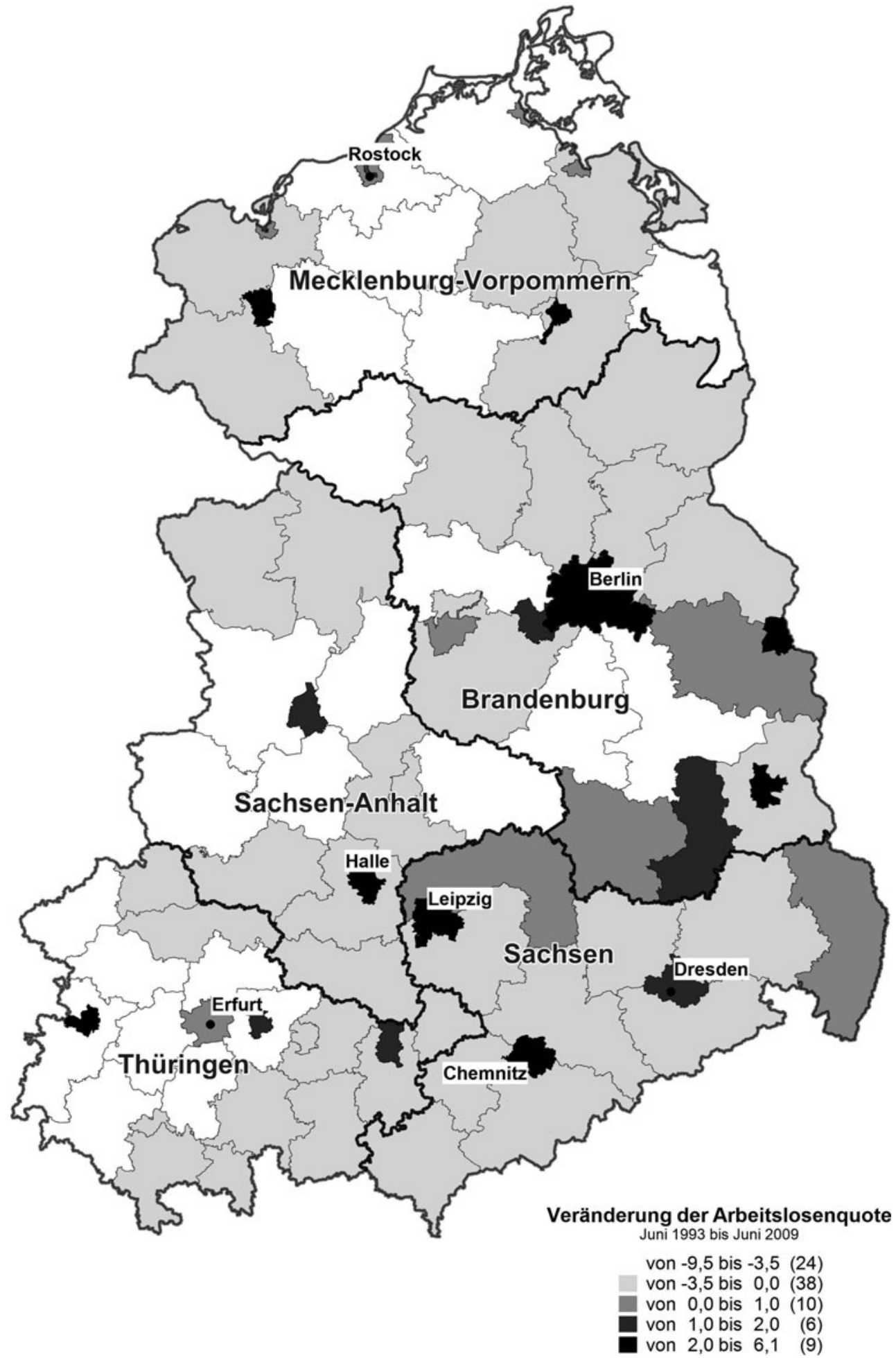

das umgebende Land. In Abb. 1 für den aktuellen Stand der Arbeitslosigkeit in Ostdeutschland findet sich die angedeutete Ringstruktur ebenfalls. Dort wird sichtbar, dass hohe Arbeitslosenquoten in Ostdeutschland besonders in ländlichen, dünn besiedelten Gebieten auftreten. Dies steht vollständig im Einklang mit den schon angesprochenen Ideen der New Economic Geography. Die Zuwächse bei den Werten für die Metropolen haben aber (noch) nicht dazu geführt, dass die Landregionen übertroffen werden. 
Abb. 6 Veränderungsrate der Arbeitslosenquote 1993-2009

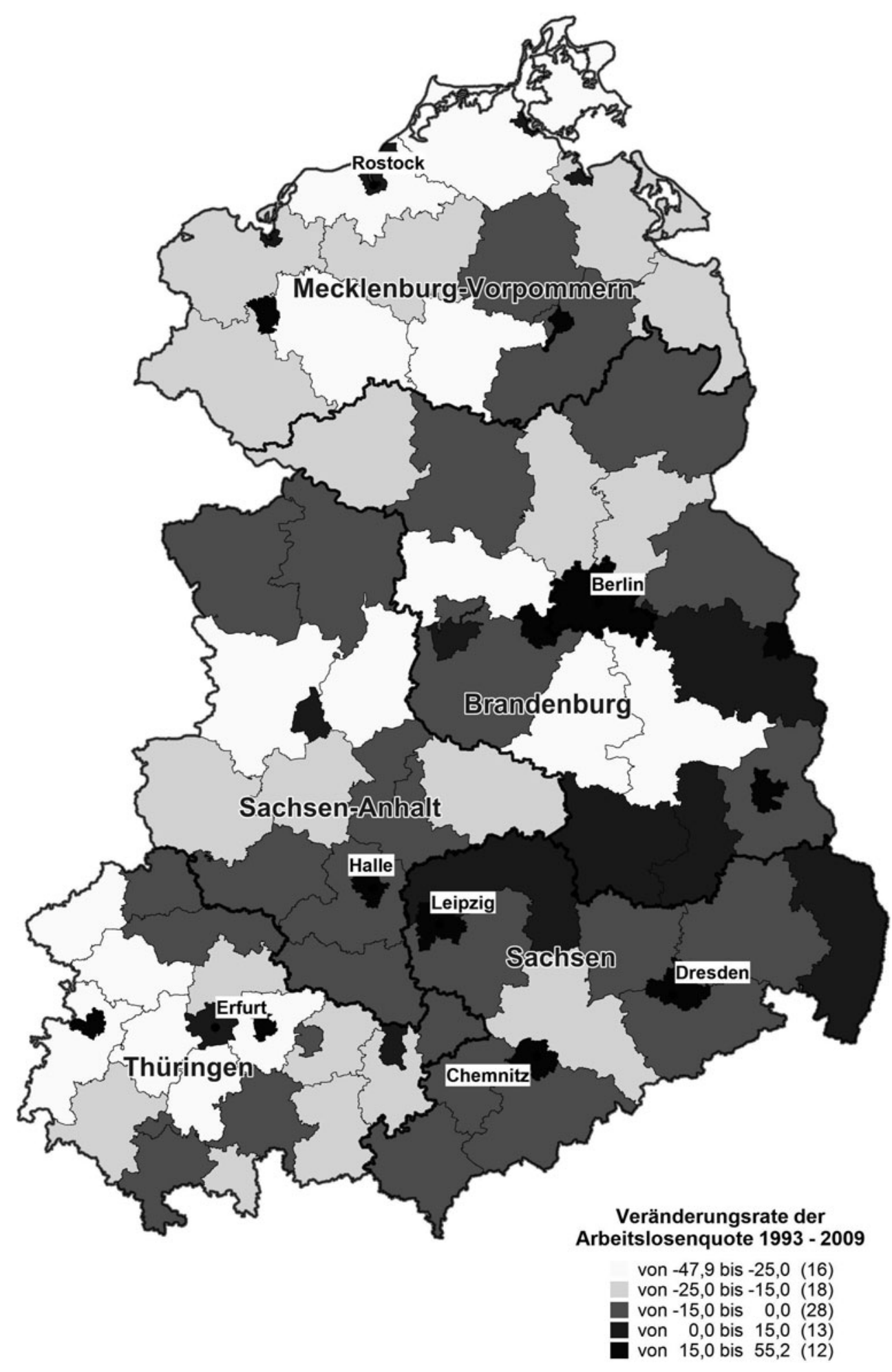

Nimmt man die Standardabweichung als Maß, variieren die Arbeitslosenquoten in Ostdeutschland ungefähr gleich stark wie im Westen. Der Wert für den Osten hat von 1993 bis 2009 von 3,5 auf 2,8 abgenommen, sodass sich eine schwache Tendenz zur Konvergenz zeigt. Die Werte für den Variationskoeffizient (Standardabweichung durch Gesamtwert), der ebenfalls zur Messung herangezogen werden kann, zeigen ein entsprechendes Bild, da die Arbeitslosigkeit in beiden Jahren fast die gleiche Höhe erreicht (Abb. 2). 
Abb. 7 Veränderung der Arbeitslosenquote 11/2008-11/2009

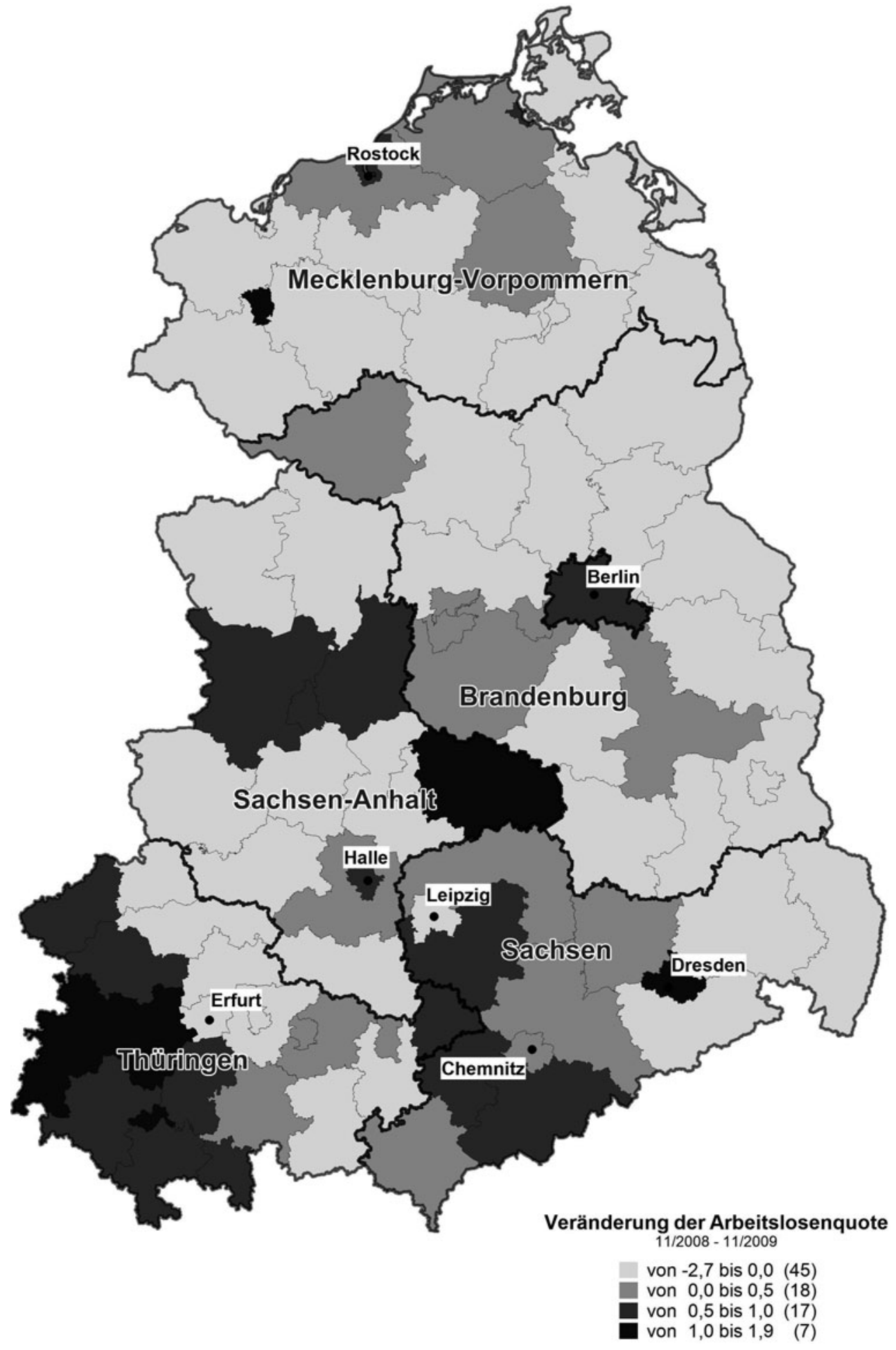

4 Räumliche Ökonometrie der Arbeitslosigkeitsentwicklung

Für die weitere Analyse der regionalen Arbeitslosigkeit innerhalb von Ostdeutschland erscheint es als angemessen, multivariate ökonometrische Modelle heranzuziehen. Dies geschieht in der Absicht, einerseits Standardansätze der Arbeitsmarktökonomie, andererseits die bereits eingeführten theoretische Überlegungen zu Agglomerationseffekten empirisch zu testen. Zu ersterem ist Elhorst (2003) hilfreich. Er 
gibt einen Überblick zu den Ansätzen der Modellierung und der zugrunde liegenden theoretischen Basis (siehe Kasten 2, zur Theorie siehe im Übrigen auch Zenou 2009).

\section{Kasten 2}

\section{Das Modell von Blanchard u. Katz (1992) in der Erweiterung von Elhorst (2003)}

Der einflussreiche Aufsatz von Blanchard u. Katz (1992) enthält mehrere Modelle, die eine regionale Anwendung von Standardansätzen der Arbeitsmarktökonomie bilden und darum für die hier wichtige Modellierung einen Hintergrund bilden können. Einer von diesen wird einerseits theoretisch formuliert, andererseits empirisch getestet. Elhorst (2003, S. 720) bezeichnet dieses Modell als das ,extensivste“, das zurzeit für empirische Analysen verfügbar ist. Er erweitert es noch geringfügig und erhält auf diese Weise jene Version, die im Folgenden dargestellt wird. Das Modell besteht aus vier Grundgleichungen, von denen die erste die Nachfrage auf dem Arbeitsmarkt beschreibt:

$$
w_{r t}=-a\left(L_{r t}-u_{r t}\right)+z_{r t} .
$$

Hier ist $w$ der Logarithmus des Lohns, $L$ ist der Log des Arbeitsangebots, $u$ die Arbeitslosenquote, $z$ gibt langfristige Effekte auf die Nachfrage wieder, $r$ ist der Index für die Region und $t$ der für die Zeit. Die zweite Gleichung ist eine Lohnkurve:

$$
w_{r t}=-f u_{r t}+X_{r}^{W} .
$$

$X^{W}$ sind hier strukturelle Einflüsse für die Lohnbildung (analog im folgenden $X^{S}$ und $X^{D}$ ). Die dritte Gleichung beschreibt das Arbeitsangebot:

$$
L_{r(t+1)}-L_{r t}=c w_{r t}-g u_{r t}+X_{r}^{S}+\varepsilon_{r t}^{S} .
$$

$\epsilon^{S}$ steht hier für exogene Schocks auf das Angebot. Langfristige Nachfrageänderungen werden gegeben durch:

$$
z_{r(t+1)}-z_{r t}=-d w_{r t}-k u_{r t}+X_{r}^{D}+\varepsilon_{r t}^{D} .
$$

Die Modellparameter werden durch $a, f, c, d, g$, und $k$ angegeben, die alle größer Null sind. Trifft man eine Gleichgewichtsannahme, die impliziert, dass die Arbeitslosenquote in der Zeit stabil bleibt und dass die Effekte externer Schocks abgeklungen sind, so ergibt sich folgender Ausdruck für die gleichgewichtige Arbeitslosenquote:

$$
u_{r}^{*}=\frac{a X_{r}^{S}+X_{r}^{D}+(a c+d) X_{r}^{W}}{a(f c+g)+f d-k} .
$$

Die Analyse macht deutlich, dass Unterschiede in der Arbeitslosigkeit langfristig Bestand haben können. Bei der empirischen Umsetzung werden allerdings hohe Ansprüche an die Beschaffbarkeit von Daten gestellt. Sind diese nicht verfügbar, ist das Modell nicht identifizierbar. Ein anderes Problem ist die Konzentration auf Gleichgewichtszustände, da man nicht davon ausgehen kann, dass die hohen Arbeitslosenquoten Ostdeutschlands bereits den Endpunkt der Entwicklung darstellen. Stattdessen wird das Modell als Analyseinstrument betrachtet, das hilft, wichtige Faktoren zu erkennen.

Da unser Thema die Entwicklung der neuen Länder während der zwei Jahrzehnte seit dem Mauerfall ist, konzentrieren wir uns auf eine Analyse von Wachstumsraten der regionalen Arbeitslosigkeit. Wir müssen noch sehen, ob die Regionen eher Persistenz zeigen oder eine Variation, ein „Wechselspiel“ in ihrer Entwicklung. Die Abb. 1 und 4 geben jeweils einen Querschnitt für den Anfangs- und den Endzeitpunkt des im Folgenden betrachteten Zeitraums von Juni 1993 bis Juni 2009 wieder. Ein Vergleich der Abbildungen zeigt bereits optisch eine bestimmte Ähnlichkeit in der räumlichen Struktur; die Korrelation der regionalen Arbeitslosenquoten beläuft sich auf 0,51. Es gibt also ein hohes $\mathrm{Ma}$ an Persistenz in der regionalen Arbeitslosigkeit, selbst in einer Transformationsökonomie über einen derartig langen Zeitraum hinweg.

Bei unserem Vorhaben der empirischen Modellierung von regionalen Längsschnitten ergeben sich für Ostdeutschland im Allgemeinen schwierige Probleme: Eine Serie von Gebietsreformen macht die Beschaffung von Daten auf regionaler Ebene schwierig und die direkte Anwendung des Modells von Elhorst (2003) unmöglich. Selbst die Arbeitslosigkeit musste auf den aktuellen Gebietsstand umgeschätzt werden, was aber mit den Informationen der BA relativ genau möglich war. Für andere Variablen ist dies nicht so leicht erreichbar. Darum wird nicht angestrebt, eine Panelschätzung durchzuführen, sondern ein Modell für das Wachstum über eine längere Zeitperiode. Aus diesen Gründen muss das zu schätzende Modell angepasst werden. Es soll außerdem Agglomerationseffekte abbilden.

Wegen des hohen Defizits an Beschäftigungsmöglichkeiten in Ostdeutschland wählen wir einen Ansatz, der von der Annahme ausgeht, dass die Arbeitslosigkeit primär durch die Nachfrageseite auf dem Arbeitsmarkt geprägt wird. Für das zu schätzende Modell wird folgende Parametrisierung verwendet:

$$
g a_{r}=\beta^{0}+\beta^{a} a_{r}+\beta^{v} v_{r}+\beta^{b} b_{r}+X_{r} \beta^{x}+\varepsilon_{r} .
$$

In Gl. 1 ist $g a_{\mathrm{r}}$ die Wachstumsrate der Arbeitslosigkeit von 1993 bis 2009, $a_{r}$ ist das Niveau der regionalen Arbeitslosigkeit im Jahre 1993, $r$ ist ein Regionsindex, er symbolisiert die 87 Landkreise und kreisfreien Städte nach 
aktueller Gebietsgliederung. Die Variable $b_{r}$ ist das Wachstum der Beschäftigten über alle Branchen hinweg und damit ein Proxy für einen der Einflüsse auf die Arbeitskräftenachfrage, wie im Kasten 2. Ebenso die Variable $v_{r}$, die den Anteil der Beschäftigten im verarbeitenden Gewerbe für das Jahr 1993 angibt. Letzteres muss erläutert werden: Unmittelbar nach der deutschen Vereinigung durchlief Ostdeutschland einen massiven Deindustrialisierungsprozess. War dieser im Jahre 1993 bereits abgeschlossen oder setzte er sich weiter fort? Stellten die im Jahre 1993 verbleibenden industriellen Bereiche Kristallisationskerne für neue Beschäftigung dar, trugen sie zur Vermeidung von Arbeitslosigkeit bei? Dies sind Fragen, für die aus der Analyse Antworten gewonnen werden können.

Die Aufnahme des Beschäftigungswachstums vermag zu beleuchten, inwieweit die Erwartung zutrifft, dass die Arbeitslosigkeit tatsächlich durch die Nachfrageseite getrieben wird. Die Beschäftigung ihrerseits war in früheren Projekten des IAB intensiv analysiert worden. Im Rahmen des schon genannten ENDOR- und des späteren VALA-Projekts (vgl. dazu u. a. Ludsteck 2006) waren die Determinanten der Beschäftigungsentwicklung untersucht worden. Im vorliegenden Kontext dient das Beschäftigungswachstum jedoch als unabhängige Variable.

$X_{r}$ ist ein Vektor an kontrollierenden (binären) Indikatorvariablen, $\epsilon_{r}$ der übliche Fehlerterm. Unter den Komponenten von $X$ befinden sich die Regionstypen nach einer verbreiteten Klassifizierung, mit denen u. a. Metropolregionen vom flachen Land unterschieden werden. Die Typen sind durch die Überkreuzung zweier Merkmale gewonnen worden, nämlich der Zentralität und der Bevölkerungsdichte (Tab. 1) und sollen Inhomogenitäten des Raumes abfangen.

Die Unterschiede zwischen den Gebietstypen sind wichtig, um Agglomerationseffekte feststellen zu können und den weiteren Bezug zur Theorie herzustellen. Sind die Erwartungen der New Economic Geography über die Zeit der Wende hinaus zutreffend, müssten sich ungünstige Arbeitsmarktlagen in den dünn besiedelten ländlichen Gebieten weitab von den Zentren feststellen lassen. Die Bedeutung von Agglomerationseffekten wurde vor allem seit Henderson et al. (1995), Glaeser et al. (1992) und Ellison u. Glaeser (1997) intensiv untersucht. Analysen für den Arbeitsmarkt Westdeutschlands finden sich u. a. bei Blien et al. (2006) und Dauth (2010).

Eine Indikatorvariable zeigt eine räumliche Lage in der Nachbarschaft zum Westen an. Es ist zu erwarten, dass Personen, die nahe an den Regionen des Westens wohnen, Beschäftigungsmöglichkeiten außerhalb Ostdeutschlands im Wege des Auspendelns nutzen. Die Arbeitslosigkeit in diesen Übergangsregionen sollte also niedriger sein als sonst $\mathrm{zu}$ erwarten wäre. Eine weitere Indikatorvariable zeigt eine Randlage zum Ausland, die eine eher höhere Arbeitslosigkeit erwarten lässt.

Zusätzlich wurden auch Testrechnungen mit dem Lohn als exogener Variablen unternommen. Allerdings macht das im Kasten 2 dargestellte theoretische Modell deutlich, dass hier die Richtung der Kausalität nicht eindeutig ist. Relativ hohe Löhne können die Folge niedriger Arbeitslosigkeit sein, sie können aber auch relativ hohe Arbeitslosenquoten zur Folge haben. Wegen diesem Endogenitätsproblem können Regressionen mit der Lohnentwicklung als unabhängiger Variablen nicht kausal interpretiert werden. Nach dem Muster der Analysen von Barro u. Sala-i-Martin (1991) wird außerdem das Wachstum der Arbeitslosigkeit auf ihr Niveau regressiert (vgl. Kasten 1).

In der Umsetzung der Analysen in einem ökonometrischen Modell stehen wir vor der Schwierigkeit, dass die einzelnen Regionen mutmaßlich nicht unabhängig voneinander sind. Es könnte sein, dass positive Impulse der Wirtschaftsentwicklung sich im Raum ausbreiten und so Regionen mit relativ niedriger Arbeitslosigkeit nebeneinander liegen. Pendelverflechtungen tragen ebenfalls zum Ausgleich von Arbeitslosenquoten zwischen benachbarten Regionen bei. All dies würde eine positive Korrelation der Arbeitslosigkeit im Raum bewirken. In die umgekehrte Richtung wirkt die Konzentration von Arbeitslosigkeit, etwa wenn bestimmte Orte Arbeitslose ,anziehen“. Dies kann zum Beispiel eintreten, wenn sogenannte „Problemgruppen“ mit einem überdurchschnittlichen Arbeitslosigkeitsrisiko bevorzugt an bestimmten Plätzen zu finden sind. In diesem Fall könnte es sein,
Tabelle 1 Klassifizierung von Regionen nach BBR (ursprünglich Görmar u. Irmen 1991)

\begin{tabular}{lll}
\hline Regionstyp 1 & Kernstädte & in Regionen mit großen Verdichtungsräumen \\
Regionstyp 2 & Hochverdichtete Kreise & \\
Regionstyp 3 & Verdichtete Kreise & \\
Regionstyp 4 & Ländliche Kreise & \\
Regionstyp 5 & Kernstädte & in Regionen mit Verdichtungsansätzen \\
Regionstyp 6 & Verdichtete Kreise & \\
Regionstyp 7 & Ländliche Kreise & in ländlich geprägten Regionen \\
Regionstyp 8 & Verdichtete Kreise & \\
Regionstyp 0 & Ländliche Kreise & \\
\hline
\end{tabular}

Der Regionstyp 2 ist in den neuen Ländern nicht präsent, der Regionstyp 0 dient in den Regressionen als Basiskategorie. 
dass man eine negative räumliche Korrelation der Arbeitslosigkeit beobachtet.

Da die hier analysierten Regionen vergleichsweise klein sind und Effekte der beschriebenen Art auftreten können, ist zumindest nicht auszuschließen, dass die Arbeitslosigkeit im Ort A in Beziehung zu jener im Nachbarort B steht. Derartige Abhängigkeitsbeziehungen verletzen jedoch die Annahmen des klassischen linearen Modells. Natürlich wäre es möglich, zu einer Betrachtung von größeren, eher geschlossenen Regionen überzugehen, dies würde aber viel Information verschenken. Abhilfe in diesem Dilemma können Methoden der räumlichen Ökonometrie schaffen, die in den vergangenen Jahren mehr und mehr aufgekommen sind und für die Cliff u. Ord (1973) und Anselin (1988) Pionierarbeiten geleistet haben. Sie haben Techniken entwickelt, die es erlauben, Modelle mit räumlicher Autokorrelation durch Verfahren des Maximum Likelihood oder mit GMM (Generalised Methods of Moments) zu schätzen. Zwei verschiedene Klassen von Modellen erweisen sich als zweckmäßig. Die erste der beiden Klassen betrifft die Einbeziehung von „Spatial Error“, d. h. von räumlichen Abhängigkeiten im Fehlerterm der Regression:

$g a=\beta^{0}+\beta^{a} a+\beta^{v} v+\beta^{b} b+X \beta^{x}+\tau \quad$ mit: $\quad \tau=\psi \mathrm{W} \tau+\varepsilon$.

In Gl. 2 sind die Variablen nicht mehr mit $r$ indiziert, sondern als Vektoren bzw. Matrizen dargestellt. In der neuen Modellierung hängt der Fehlerterm $\tau_{r}$ des Regressionsmodells für eine konkrete Region vom Fehler in Nachbarregionen ab. Die Matrix W nimmt die Beziehungen der Regionen untereinander auf. Im vorliegenden Fall spezifizieren wir einfach eine Nachbarschaftsmatrix: Sie enthält für Regionen, die direkte Nachbarn sind, eine Eins, für alle anderen Regionen jeweils eine Null. Zur konkreten Umsetzung wird die Matrix wie in der Literatur üblich so normiert, dass die Zeilensummen sich zu 1 addieren. Ist die Gl. 2 das maßgebliche Modell, so werden die Koeffizienten mit der Pooled Regression von Gl. 1 ineffizient und die Standardfehler verzerrt geschätzt. Ein weiteres relevantes Modell enthält nun keinen Spatial Error, sondern einen „Spatial Lag“:

$$
g a=\beta^{0}+\phi \mathrm{W} g a+\beta^{a} a+\beta^{v} v+\beta^{b} b+X \beta^{x}+\varepsilon .
$$

Hier beeinflusst das Wachstum der Arbeitslosigkeit $g a_{r}$ der Region $r$ das Wachstum der Nachbarregionen, erneut definiert über die Matrix W. Gilt Gl. 3 und wird stattdessen die Pooled Regression (Gl. 1) verwendet, werden alle Koeffizienten verzerrt geschätzt. Im Folgenden werden zur Schätzung der Modelle Verfahren des Maximum Likelihood nach Anselin (1988) und Anselin u. Hudak (1992) verwendet.

Für die in den Ergebnistabellen zusätzlich angegebenen LM-Tests sind Florax u. Nijkamp (2003) sowie Anselin et al. (1996) maßgeblich. Diese Tests dienen der Modellauswahl, denn sie zeigen an, welche Art von räumlicher
Abhängigkeit vorliegt. Sind sowohl Spatial Lag als auch Spatial Error signifikant, wird üblicherweise das Modell mit dem höheren Signifikanzniveau bevorzugt. Vom LM-Test gibt es zwei Versionen, eine „einfache“ und eine „robuste“ Variante. Die bevorzugte robuste berücksichtigt zusätzlich, dass neben einem Spatial Lag auch ein Spatial-Error-Modell zutreffen könnte (und umgekehrt).

Die Tab. 2 und 3 sowie Tab. 4 und 5 im Anhang zeigen Ergebnisse von Regressionsanalysen für die Entwicklung der Arbeitslosigkeit. Zwei verschiedene Gruppen von Modellen wurden gerechnet: einmal eine mit der Beschäftigungsentwicklung als unabhängiger Variablen (Modelle 4-6) und einmal eine Gruppe ohne diese (Modelle 1-3). In jeder der beiden Gruppen wurde eine Pooled Regression, ein Modell mit räumlichem Fehlerterm und ein Modell mit räumlichem Lag geschätzt.

Die Tests zeigen ein relativ konsistentes Bild: Das Modell mit räumlichem Lag wird gegenüber der Pooled Regression und dem Modell mit räumlichem Fehlerterm bevorzugt. Allerdings ist auch im Modell 5 der räumliche Autokorrelationskoeffizient signifikant. Interessanterweise ist dieser Koeffizient durchgängig negativ. Das gleiche gilt für den Koeffizienten der räumlich verzögerten Variablen. Dies ist ein relativ seltenes Ergebnis, bedeutet es doch, dass die Entwicklung der Arbeitslosigkeit negativ mit der Entwicklung an Nachbarorten verbunden ist. Man könnte vermuten, dass dieser Effekt auf Suburbanisierungsphänomene und dergleichen zurückgeführt werden kann. Derartige Entwicklungen müssten aber von den Regionaltypen aufgenommen werden. Stattdessen ist der negative Wert ein sehr wichtiges statistisches Spiegelbild der optisch, anhand der Kartendarstellungen festgestellten angedeuteten Kreisstruktur: Regionen mit relativ niedrigem Arbeitslosigkeitswachstum liegen tendenziell neben solchen mit niedriger Veränderung.

Es wurde schon ausgeführt, dass eine derartige negative räumliche Autokorrelation nicht sehr häufig vorkommt. In dem wichtigen Aufsatz von Anselin et al. (1996) wird dieser Fall nicht behandelt. In diesem Aufsatz ist der LM-Test immer konsistent mit dem Test des Parameters zur räumlichen Autokorrelation (also für $\lambda$ bzw. $\rho$ ), der direkt dem Modell entnommen wird. In unserem Kontext ergeben sich zwar keine großen Abweichungen, aber doch unterschiedliche Signifikanzniveaus, z. B. im Fall des Modells 2, in dem der robuste LM-Test eine hohe Signifikanz anzeigt, aber der Test für $\lambda=0$ im Modell selbst nicht. In uneindeutigen Fällen ist nicht klar, welchem Test der Vorzug zu geben ist. Aus diesem Grund wird hier zusätzlich der Wald-Test angegeben und neben dem robusten auch der einfache LM-Test. An dieser Stelle ist zweifellos Raum für weitere methodische Untersuchungen, deren Ergebnisse hier nicht vorweggenommen werden können. Immerhin stimmen die Tests soweit überein, dass die vorliegenden Ergebnisse für die Bedeutung von regionalen Effekten spre- 
chen. Da das Modell mit räumlichen Lags präferiert wird, muss die Pooled Regression als fehlspezifiziert aufgefasst werden.

Tabelle 3 stellt einige weitere Ergebnisse für Modelle zusammen, bei denen die nicht signifikanten Regionstypen eliminiert wurden. Das Ergebnis für den Gebietstyp 1 (Kernstadt) ist nun als relativ zu allen anderen Gebietstypen $\mathrm{zu}$ interpretieren. Inhaltlich ergibt sich in den Modellen 7-9 kaum eine Veränderung der bereits in Tab. 2 gefundenen Ergebnisse. In den Modellen 10-12 wurde die Variable „Nachbar Ausland“ zusätzlich einbezogen, die jedoch wiederum nicht signifikant ausfällt.
Das Niveau der Arbeitslosigkeit von 1993 hat einen negativen Koeffizienten. Diese sogenannte $\beta$-Konvergenz ist jedoch nur ein notwendiger, aber kein hinreichender Beleg für die Abnahme der Streuung der Arbeitslosigkeit zwischen Regionen, d. h. von $\sigma$-Konvergenz (siehe Kasten 1). Zusammen mit der bereits genannten geringen Abnahme der Standardabweichung ergibt sich jedoch eine schwache Tendenz zur Konvergenz. Die Regionen der östlichen Länder gleichen sich im Hinblick auf die Höhe der Arbeitslosigkeit ein wenig aneinander an.

Höhere Anteile des verarbeitenden Gewerbes reduzieren tendenziell die Arbeitslosigkeit. Die Hypothese scheint sich

Tabelle 2 Ergebnisse für die Entwicklung der Arbeitslosigkeit 1993-2009 ( $t$ - bzw. $z$-Werte in Klammern)

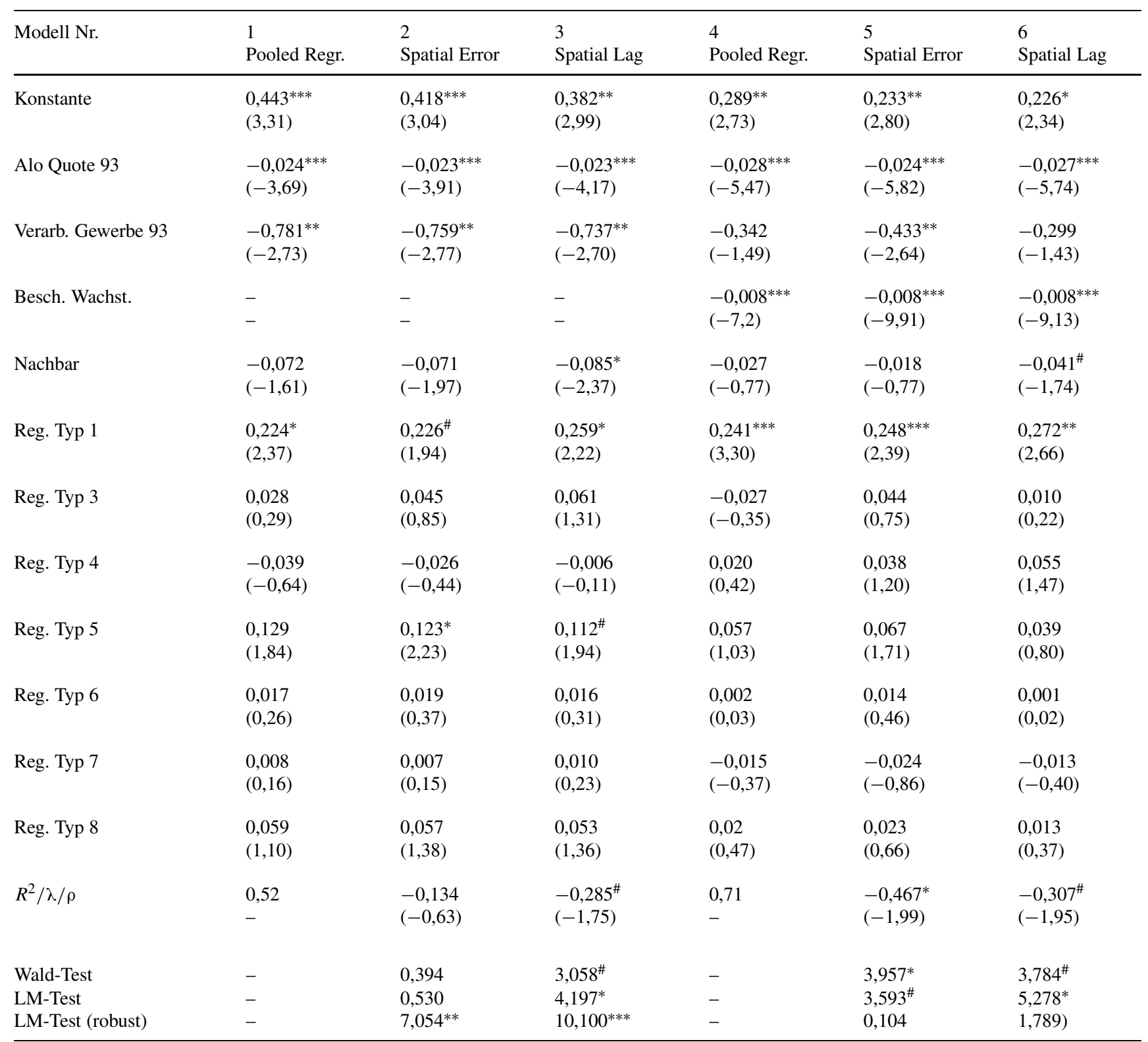

*** $0,001,{ }^{* *} 0,01,{ }^{*} 0,05,{ }^{\#} 0,10 \%$ Niveau signifikant.

Daten: Arbeitslosenstatistik der BA, Beschäftigungsstatistik der BA, Gebietsstandsbereinigung IAB. 
Tabelle 3 Ergebnisse für die Entwicklung der Arbeitslosigkeit 1993-2009 (II)

\begin{tabular}{|c|c|c|c|c|c|c|}
\hline $\begin{array}{l}\text { Modell Nr. } \\
\text { Variable }\end{array}$ & $\begin{array}{l}7 \\
\text { Pooled Regr. }\end{array}$ & $\begin{array}{l}8 \\
\text { Spatial Error }\end{array}$ & $\begin{array}{l}9 \\
\text { Spatial Lag }\end{array}$ & $\begin{array}{l}10 \\
\text { Pooled Regr. }\end{array}$ & $\begin{array}{l}11 \\
\text { Spatial Error }\end{array}$ & $\begin{array}{l}12 \\
\text { Spatial Lag }\end{array}$ \\
\hline Konstante & $\begin{array}{l}0,530^{* * *} \\
(5,15)\end{array}$ & $\begin{array}{l}0,484^{* * *} \\
(3,74)\end{array}$ & $\begin{array}{l}0,458^{* * *} \\
(3,39)\end{array}$ & $\begin{array}{l}0,530^{* * *} \\
(5,12)\end{array}$ & $\begin{array}{l}0,472^{* * *} \\
(3,43)\end{array}$ & $\begin{array}{l}0,447^{* * *} \\
(3,79)\end{array}$ \\
\hline Arbeitslosenquote 1993 & $\begin{array}{l}-0,028^{* * *} \\
(-5,16)\end{array}$ & $\begin{array}{l}-0,024^{* * *} \\
(-4,90)\end{array}$ & $\begin{array}{l}-0,027^{* * *} \\
(-5,33)\end{array}$ & $\begin{array}{l}-0,028^{* * *} \\
(-5,11)\end{array}$ & $\begin{array}{l}-0,026^{* * *} \\
(-4,88)\end{array}$ & $\begin{array}{l}-0,027^{* * *} \\
(-5,53)\end{array}$ \\
\hline Anteil verarb. Gewerbe 1993 & $\begin{array}{l}-0,802^{* * *} \\
(-3,38)\end{array}$ & $\begin{array}{l}-0,707^{* *} \\
(-2,64)\end{array}$ & $\begin{array}{l}-0,725^{* *} \\
(-3,50)\end{array}$ & $\begin{array}{l}-0,802^{* * *} \\
(-3,36)\end{array}$ & $\begin{array}{l}-0,687^{*} \\
(-2,47)\end{array}$ & $\begin{array}{l}-0,719^{* *} \\
(-3,01)\end{array}$ \\
\hline Nachbar West & $\begin{array}{l}-0,087^{*} \\
(-2,27)\end{array}$ & $\begin{array}{l}-0,085^{\text {*** }} \\
(-2,16)\end{array}$ & $\begin{array}{l}-0,102^{* * *} \\
(-3,50)\end{array}$ & $\begin{array}{l}-0,087^{*} \\
(-2,23)\end{array}$ & $\begin{array}{l}-0,023 \\
(-1,49)\end{array}$ & $\begin{array}{l}-0,098^{* * *} \\
(-3,32)\end{array}$ \\
\hline Nachbar Ausland & $\begin{array}{l}- \\
-\end{array}$ & $\begin{array}{l}- \\
-\end{array}$ & $\begin{array}{l}- \\
-\end{array}$ & $\begin{array}{l}0,001 \\
(0,03)\end{array}$ & $\begin{array}{l}0,023 \\
(0,55)\end{array}$ & $\begin{array}{l}0,039 \\
(1,00)\end{array}$ \\
\hline Regionstyp 1 & $\begin{array}{l}0,177^{*} \\
(2,17)\end{array}$ & $\begin{array}{l}0,171 \\
(1,46)\end{array}$ & $\begin{array}{l}0,208^{\#} \\
(1,83)\end{array}$ & $\begin{array}{l}0,176^{*} \\
(2,15)\end{array}$ & $\begin{array}{l}0,174 \\
(1,49)\end{array}$ & $\begin{array}{l}0,218^{\#} \\
(1,91)\end{array}$ \\
\hline$R^{2} / \psi / \varphi$ & $\begin{array}{l}0,48 \\
-\end{array}$ & $\begin{array}{l}-0,205 \\
(-0,98)\end{array}$ & $\begin{array}{l}-0,335^{*} \\
(-2,41)\end{array}$ & $\begin{array}{l}0,48 \\
-\end{array}$ & $\begin{array}{l}0,249 \\
(-0,96)\end{array}$ & $\begin{array}{l}-0,381^{*} \\
(-2,50)\end{array}$ \\
\hline $\begin{array}{l}\text { Wald-Test } \\
\text { LM-Test } \\
\text { LM-Test (robust) }\end{array}$ & $\begin{array}{l}- \\
- \\
-\end{array}$ & $\begin{array}{l}0,961 \\
1,364 \\
3,461^{\#}\end{array}$ & $\begin{array}{l}5,824^{*} \\
6,193^{*} \\
8,290^{* *}\end{array}$ & $\begin{array}{l}- \\
- \\
-\end{array}$ & $\begin{array}{l}0,921 \\
1,332 \\
4,349\end{array}$ & $\begin{array}{l}6,245^{*} \\
6,327^{* *} \\
9,343^{* *}\end{array}$ \\
\hline
\end{tabular}

${ }^{* * *} 0,001,{ }^{* *} 0,01,{ }^{*} 0,05,{ }^{\#} 0,10 \%$ Niveau signifikant.

Daten: Arbeitslosenstatistik der BA, Beschäftigungsstatistik der BA, Gebietsstandsbereinigung IAB. Regionstypen 2-9 nicht einbezogen.

tatsächlich zu bestätigen, da an Orten, wo sich Kristallisationskerne für das verarbeitende Gewerbe finden, eine eher positive Arbeitsmarktentwicklung stattfindet. Hier ist es anscheinend leichter möglich, weitere Betriebe zu etablieren, die Beschäftigung schaffen und die Arbeitslosigkeit reduzieren. Dies ist ein Beleg für das Auftreten von Agglomerationseffekten. In den Modellen 1-3 wirkt sich die Randlage zum Westen positiv aus. Schon deskriptive Analysen zeigen, dass die Arbeitslosenquoten in derartigen Regionen eher niedriger ausfallen.

Die Modelle 4-6 zeigen, dass das Beschäftigungswachstum die Entwicklung der Arbeitslosigkeit sehr stark beeinflusst, die zusätzliche Aufnahme dieser Variablen erhöht das $R^{2}$ der Pooled Regression von 52 auf $71 \%$. Die Beschäftigungsentwicklung ist also wie erwartet prägend für die Entwicklung der Arbeitslosigkeit. Die Einflüsse anderer Variablen sind dann unter Umständen nicht mehr signifikant, wenn die Beschäftigungsentwicklung mit aufgenommen wird. Ein Beispiel dafür ist die Variable, die anzeigt, dass eine Regionen in Nachbarschaft zum Westen liegt. Der Effekt der Randlage ist dann schon in jenem der Beschäftigungsentwicklung enthalten.

Wichtig ist die Untersuchung auf differenzierende Wirkungen der einzelnen Gebietstypen. Es wurde schon ausgeführt, dass deskriptive Analysen zeigen, dass die Arbeitslosigkeit in den ländlichen Regionen fernab vom
Westen und von Berlin besonders hoch ist. Dies ist aber offensichtlich ein Effekt, der aus der Anfangszeit der Vereinigung ,ererbt“ ist (und durch Ansätze der New Economic Geography oder durch konkurrierende Ansätze verstanden werden kann). Aktuell kann man nur eine geringe Differenzierung der Regionstypen in der Arbeitslosigkeitsentwicklung feststellen. Es gibt allerdings eine wichtige Ausnahme. Die Kernstädte des Gebietstyps 1 verzeichnen ein deutliches Ansteigen der Arbeitslosigkeit. Dabei handelt es sich vor allem um ein Suburbanisierungsphänomen. Fasst man nämlich in weiteren Rechnungen (nicht in den Tabellen dargestellt) die Regionstypen 1-4 zusammen, ist ihr Koeffizient nicht signifikant. Das Ansteigen der Arbeitslosigkeit in den Kernstädten ist ein Angleichungsprozess gegenüber der Arbeitsmarktsituation im Westen, wo ebenfalls in den Großstädten tendenziell überdurchschnittliche Arbeitslosenquoten auftreten.

Die Indikatorvariable für die Randlage zum Ausland ist überraschenderweise nicht signifikant. Dies mag daran liegen, dass zum Startjahr 1993 die Arbeitslosigkeit in diesen Regionen bereits überdurchschnittlich hoch war.

Einige Testrechnungen mit weiteren Variablen wurden durchgeführt, obwohl die in Tab. 4 (im Anhang) enthaltenen Ergebnisse nur deskriptiv interpretiert werden können. Dies gilt für die Lohnentwicklung, bei der ein Einfluss auf die Arbeitslosigkeit einer Abhängigkeit von dieser gegenü- 
bersteht („Lohnkurveneffekt“, vgl. Gl. 2' im Kasten 2). Es gilt auch für die Bevölkerungsentwicklung. Wie das im Kasten dargestellte Modell deutlich macht, würde man erwarten, dass eine Abwanderung aus einer Region das Arbeitskräftepotenzial reduziert und die Arbeitslosenquote dadurch senkt. Da die Abwanderung jedoch vor allem aus Regionen erfolgen wird, in denen die Arbeitslosigkeit überdurchschnittlich ist, registriert das Modell einen entgegenwirkenden Effekt. Andererseits hat unsere kurze Diskussion der New Economic Geography bereits gezeigt, dass die arbeitsmarktbedingte Abwanderung aus Regionen die Arbeitslosigkeit weiter erhöhen kann. Um die beschriebenen Endogenitätsprobleme zu vermeiden, werden diese Modelle hier nicht kausal interpretiert.

Tabelle 5 enthält eine Zusammenstellung von Modellen mit einer Variation einer der zentralen Variablen. Hier wurde das verarbeitende Gewerbe enger definiert, indem nur sogenannte ,Zukunftsbranchen“ aufgenommen wurden, z. B. Elektronik, Kfz, Optik und andere mehr, während Branchen, die im Strukturwandel an Bedeutung verlieren, wie z. B. Textil etc., ausgeschlossen wurden. Substanziell ergeben sich die gleichen Ergebnisse wie in Tab. 2, die Wirkung des verarbeitenden Gewerbes fällt jedoch in dieser Variante deutlicher aus. Damit wird die Hypothese erneut bestätigt, dass das verarbeitende Gewerbe einen Kristallisationskern für die regionale Entwicklung darstellt.

Letzterer Aspekt ist von allgemeiner Bedeutung: Die Ergebnisse zeigen, dass Deindustrialisierung für Regionalentwicklung und Arbeitsmarkt gefährlich ist, weil das verarbeitende Gewerbe unverzichtbare Impulse für die Stabilisierung der Beschäftigung bietet. Die quantitative Analyse hat damit wichtige Ergebnisse (nicht nur) für die Struktur regionaler Disparitäten auf den Arbeitsmärkten Ostdeutschlands erbracht. Zusammenfassend können wir anmerken, dass eine schwache Tendenz zur Konvergenz sichtbar wurde. Außerdem weisen Regionen mit einer Nachbarschaft zum Westen eine eher günstige Entwicklung der Arbeitslosenquoten auf. Der Verdacht auf unvorteilhafte Werte in Räumen mit einer Randlage zum Ausland bestätigt sich nicht. Und schließlich wächst die Arbeitslosenquote überproportional in den großen Städten.

\section{Aktuelle Lage und Entwicklungsperspektiven}

Die aktuelle schwere Konjunkturkrise beeinträchtigt auch Ostdeutschland, allerdings bisher in geringerem Grade als den Westen. Die Arbeitslosenquote Ost sinkt immer noch - von 13,7\% im November 2008 auf 13,2\% im November 2009 - während jene für den Westen ansteigt. Wegen der Intensität der Krise ist dies ein außerordentlich erstaunliches Ergebnis. Sieht man sich die regionale Verteilung der absoluten Veränderungen innerhalb Ostdeutschlands an, so zeigt sich tendenziell ein Ost-West-Gefälle (vgl. Abb. 7 für einen aktuellen Überblick), das einige Monate vorher noch ausgeprägter war. Überraschenderweise liegen die Regionen mit Abnahmen der Arbeitslosigkeit eher im Osten, während im Westen die Arbeitslosigkeit häufiger ansteigt. Der Grund dafür ist, dass die Krise vor allem die erfolgreichen, exportorientierten Regionen trifft, die sich eher im Westen finden. Die interregionalen Verflechtungen innerhalb der deutschen Wirtschaft sind jedoch so stark, dass keine Region von der Krise vollständig verschont bleibt. Die Kartendarstellungen machen insgesamt die Bedeutung räumlicher Verflechtungen deutlich.

Geht man von der aktuellen konjunkturellen Lage zu einer längerfristigen Betrachtung Ostdeutschlands über, so ergeben sich einige positive Perspektiven, trotz der bisher generell unbefriedigenden Lage. Die Produktivität wuchs in der jüngeren Vergangenheit deutlich schneller als im Westen. Von 1999 bis 2008 stieg das Bruttoinlandsprodukt je Erwerbstätigem im Westen um $8 \%$, aber im Osten um $20 \%$ (BMWi 2009). Dies ist eine Entwicklung, die vor allem vom verarbeitenden Gewerbe getragen wird. Sein Anteil an der Bruttowertschöpfung stieg überproportional, erreicht inzwischen fast $20 \%$, dies ist aber immer noch niedriger als der Anteil von $25 \%$ im Westen. Allerdings sind die ostdeutschen Exportquoten deutlich niedriger als jene im Westen. Dies ist u. a. darauf zurückzuführen, dass ostdeutsche Betriebe jünger und kleiner sind als westdeutsche. Das vergleichsweise hohe Produktivitätswachstum impliziert jedoch, dass derartige Defizite künftig tendenziell ausgeglichen werden.

Auffällig ist, dass die Entwicklung in Ostdeutschland wenig Impulse aus dem benachbarten Ausland bezieht. Berlin und andere Städte wie Frankfurt an der Oder erscheinen von ihrer räumlichen Lage her als prädestiniert für den Handel mit Polen und anderen osteuropäischen Ländern. Es wurde jedoch bereits gezeigt, dass sich dieser potenzielle Standortvorteil in der Praxis als Nachteil erweist. Dieser könnte u. a. damit zusammenhängen, dass die Vergangenheit der Städte nicht dazu geführt hat, leitende Funktionen von Unternehmen dort anzusiedeln. Mit der weiteren Integration vor allem von Polen und von Tschechien in die EU ist jedoch zu hoffen, dass der Osten Deutschlands zusätzliche positive Impulse für Wachstum und Beschäftigung erhält.

\section{Kurzfassung}

Die schockartige Öffnung der Ökonomie Ostdeutschlands hatte tief greifende Folgen für die Bewohner dieser Region. Die Lebensverhältnisse verbesserten sich, es entstanden aber auch massive Arbeitsmarktprobleme, die im vorliegenden Beitrag vor allem im Hinblick auf die Arbeitslosigkeit in zwei Schritten analysiert werden: Zunächst liegt der Fokus auf Ostdeutschland als Einheit, sodann wird die interne Differenzierung untersucht. 
20 Jahre nach dem Mauerfall sind Ökonomie und Arbeitsmarkt in Deutschland immer noch von einem starken West-Ost-Gefälle geprägt. In der Zeit nach der Grenzöffnung trugen schnell ansteigende Löhne zu dem Anstieg der Arbeitslosigkeit bei. Dann stagnierten die Löhne für lange Zeit und blieben deutlich niedriger als im Westen. Die ungünstigen Arbeitsmarktbedingungen waren eine wichtige Ursache für die hohe Abwanderung aus Ostdeutschland. Zwischen 1990 und 2007 betrug die (saldierte) Nettomigration 1,5 Mio. Menschen, Ostdeutschland verlor einen guten Teil seiner Bevölkerung. Trotzdem blieb für lange Zeit das Niveau der Arbeitslosigkeit bei rund $20 \%$.

Zum Verständnis dieser Situation tragen spezielle Ansätze der Neuen Ökonomischen Geografie und verwandte regionalökonomische Theorien bei, in die der Arbeitsmarkt integriert wurde. Diese Ansätze kennen die Eigenschaft der Pfadabhängigkeit und erlauben dadurch die langfristige Existenz einer Zentrum-Peripherie-Struktur. Aus der Sichtweise dieser Ansätze hat Auswanderung gegensätzliche Effekte auf die Arbeitslosigkeit: Sie wirkt auf die Höhe des Arbeitskräftepotenzials und reduziert dadurch direkt das Volumen der Beschäftigungslosen. Zum anderen verringert Auswanderung jedoch auch das Marktpotenzial der Region, d. h. die dort lokalisierte kaufkräftige Nachfrage. Dadurch kann die Arbeitslosigkeit auf indirektem Weg steigen.

In jüngerer Zeit scheint der letztgenannte Mechanismus jedoch nicht mehr wirksam zu sein, da in den vergangenen Jahren die Rate der Arbeitslosigkeit in Ostdeutschland stärker als im Westen zurückgegangen ist. Selbst in Zeiten der Krise fiel die Arbeitslosigkeit bis auf 13,2\% im November 2009.

Innerhalb Ostdeutschlands ergibt sich eine starke Differenzierung der Arbeitsmarktlagen, die über die Zeit relativ stabil bleibt. Die Entwicklung der Arbeitslosigkeit wird in einem räumlichen ökonometrischen Modell analysiert. Dabei zeigen sich schwache Konvergenzprozesse ( $\beta$ - und $\sigma$-Konvergenz), sodass Regionen mit zunächst hohen Arbeitslosenquoten später eher höhere Abnahmen aufweisen. Für die Reduktion der Arbeitslosigkeit erweisen sich Kerne des verarbeitenden Gewerbes als vorteilhaft. Die Entwicklung in den Regionen wird von jener in den Nachbargebieten beeinflusst, da räumliche Autokorrelation feststellbar ist. Bemerkenswert ist, dass diese Autokorrelation negativ ausfällt, was in vergleichbaren Analysen nur selten vorkommt. Zum Verständnis der räumlichen Differenzierung können die bereits erwähnten theoretischen Ansätze herangezogen werden.

\section{Executive summary}

For purposes of research, Eastern Germany is a very interesting case since German unification could be regarded as a "natural experiment", opening up possibilities to test various theories of regional economics among others. The analysis of this paper is carried out in two steps: The first one is based on a view at the labour market of Eastern Germany as a whole. The second one is an analysis of the internal differentiation of the East.

20 years after the fall of the Berlin Wall there are still considerable differences between the economies and labour markets of Eastern and Western Germany. Fast increases in the wage level of the East were one of the causes of high increases of the unemployment rate during the early nineties. Then wages stagnated for a long time and many people moved out of this area, migrated to prospering regions in the West. Net migration amounted to $1.5 \mathrm{Mio}$. between 1990 and 2007. Unemployment in the East remained high however (about $20 \%$ up to 2005).

The development can be understood by special approaches of New Economic Geography which integrate the labour market into the theoretical framework and by related theories. In a view of this kind outmigration has two effects on the stock of the unemployed. The direct effect is the reduction of the labour force and therefore the reduction of unemployment. There is an indirect effect, however, working in the opposite direction. It reduces the market potential of these regions and contributes to high unemployment rates. In recent times this mechanics seem to be broken. In the last few years unemployment has fallen especially in Eastern Germany (to $13.2 \%$ in November 2009), even in times of economic crisis.

Within Eastern Germany a strong variation of labour market situations is visible, which has been relatively stable over time. In detail, for unemployment a slightly blurred ring structure is showing up with Berlin as its centre. The development of unemployment is analysed in a spatial econometrics framework. This is relevant, since spatial autocorrelation is found to be significant. It is remarkable that this autocorrelation is negative.

The empirical analyses show a weak tendency towards convergence of regional unemployment rates. Several influences on the development of unemployment are taken into account. It can be seen that the industry and the qualification structure are relevant for the development of unemployment. Theoretically, the mentioned approaches of regional economics also help the understanding of regional differentiation.

Danksagung Die Autoren danken W. Dauth und K. Wolf (beide IAB) für Unterstützung bei den statistischen Analysen und den Teilnehmern am IAB/IWH-Workshop „20 Jahre Arbeitsmarkt Ostdeutschland - Erfahrungen und Perspektiven“ im Oktober 2009 für inhaltliche Anregungen. Jede Verantwortung für die Durchführung der Analysen, für die erzielten Resultate und für deren Interpretation verbleibt jedoch allein bei den Autoren. 


\section{Anhang}

Tabelle 4 Ergebnisse für die Entwicklung der Arbeitslosigkeit 1993-2009 mit ergänzenden Analysen (nur Pooled Regression)
Tabelle 5 Ergebnisse für die Entwicklung der Arbeitslosigkeit 1993-2009 mit ergänzenden Analysen: Nur „Zukunftsbranchen“ im Verarbeitenden Gewerbe

\begin{tabular}{llll}
\hline Modell Nr. & A1 & A2 & A3 \\
\hline Konstante & $0,414^{* * *}$ & 0,307 & $0,440^{* *}$ \\
& $(3,19)$ & $(1,40)$ & $(3,27)$ \\
Arbeitslosigkeit 1993 & $-0,030^{* * *}$ & $-0,024^{* * *}$ & $-0,024^{* * *}$ \\
& $(-5,75)$ & $(-3,35)$ & $(-3,70)$ \\
Verarb. Gewerbe 1993 & $-0,551^{*}$ & $-0,828^{* *}$ & $-0,773^{* *}$ \\
Lohnwachstum & $(-2,42)$ & $(-2,79)$ & $(-2,69)$ \\
Bevökerungswachstum & - & $-0,493$ & - \\
& - & $(-0,76)$ & - \\
Nachbar West & $-0,757^{* * *}$ & - & - \\
Nachbar Ausland & $(-6,91)$ & - & $-0,069$ \\
& $-0,039$ & $-0,067$ & $(-1,53)$ \\
Regionstyp 1 & $(-1,08)$ & $(-1,47)$ & 0,027 \\
& 0,009 & 0,034 & $(0,62)$ \\
$R^{2}$ & $(0,27)$ & $(0,76)$ & $0,229^{*}$ \\
\hline
\end{tabular}

*** $0,001,{ }^{* *} 0,01,{ }^{*} 0,05 \%$ Niveau signifikant.

Regionstypen 3-8 einbezogen, aber nicht dargestellt (nicht signifikant).

\begin{tabular}{llll}
\hline Modell Nr. & A4 & A5 & A6 \\
& Pooled Regr. & Spatial Error & Spatial Lag \\
\hline Konstante & $0,392^{* *}$ & $0,387^{* *}$ & $0,342^{* *}$ \\
& $(3,21)$ & $(2,97)$ & $(2,97)$ \\
Alo Quote 93 & $-0,018^{* *}$ & $-0,018^{* * *}$ & $-0,018^{* * *}$ \\
& $(-2,74)$ & $(-3,22)$ & $(-3,43)$ \\
Verarb. Gewerbe 93 & $-1,491^{* * *}$ & $-1,478^{* * *}$ & $-1,375^{* * *}$ \\
& $(-3,64)$ & $(-4,35)$ & $(-4,42)$ \\
Nachbar & $-0,041$ & $-0,041$ & $-0,054$ \\
& $(-0,90)$ & $(-1,16)$ & $(-1,59)$ \\
Reg. Typ 1 & $0,262^{* *}$ & $0,263^{*}$ & $0,283^{* *}$ \\
& $(2,87)$ & $(2,35)$ & $(2,57)$ \\
$R^{2} / \lambda / \rho$ & 0,55 & $-0,027$ & $-0,2229$ \\
& - & $(-0,12)$ & $(-1,41)$ \\
Wald-Test & - & & 1,980 \\
LM-Test & - & 0,014 & 2,338 \\
LM-Test (robust) & - & 0,018 & $11,546^{* * *}$
\end{tabular}

*** $0,001,{ }^{* *} 0,01, * 0,05 \%$ Niveau signifikant.

Regionstypen 3-8 einbezogen, aber nicht dargestellt (nicht signifikant). 


\section{Literatur}

Anselin, L.: Spatial Econometrics: Methods and Models. Kluwer Academic, Dordrecht (1988)

Anselin, L., Hudak, S.: Spatial econometrics in practice. Reg. Sci. Urban Econ. 22, 509-536 (1992)

Anselin, L., Bera, A.K., Florax, R., Yoon, M.J.: Simple diagnostic tests for spatial dependence. Reg. Sci. Urban Econ. 26, 77-104 (1996)

BMWi - Bundesministerium für Wirtschaft und Technologie (Hrsg.): Wirtschaftsdaten Neue Länder (2009)

Barro, R.J.: Eastern Germany's long haul. Wall Street J., May 3 (1991)

Barro, R.J., Sala-i-Martin, X.: Convergence across states and regions. Brookings Pap. Eco. Ac. 1, 107-182 (1991)

Barro, R.J., Sala-i-Martin, X.: Economic Growth, 2nd edn. MIT, Cambridge (Mass.), London (2004)

Blanchard, O.J., Katz, L.F.: Regional evolutions. Brookings Pap. Econ. Ac. 1, 1-75 (1992)

Blien, U., Graef, F.: Entropy optimizing methods for the estimation of tables. In: Balderjahn, I., Mathar, R., Schader, M. (Hrsg.) Classification, Data Analysis, and Data Highways, Proceedings of the 21st Annual Conference of the Gesellschaft für Klassifikation, S. 3-15. Springer, Berlin (1998)

Blien, U., Maierhofer, E., Vollkommer, D., Wolf, K.: Einflussfaktoren der Entwicklung ostdeutscher Regionen. Theorie, Daten, Deskriptionen und quantitative Analysen. In: Blien, U. (Hrsg.) Die Entwicklung der ostdeutschen Regionen. Beiträge zur Arbeitsmarktund Berufsforschung, Bd. 267, S. 67-254. Nürnberg (2003)

Blien, U., Südekum, J., Wolf, K.: Local employment growth in West Germany: a dynamic panel approach. Labour Econ. 13(4), 445-458 (2006)

Bliss, C.: Galton's fallacy and economic convergence. Oxford Econ. Pap. 51, 4-14 (1999)

Bogai, D., Hirschenauer, F.: Die Entwicklung des Arbeitsmarktes in Ostdeutschland. ifo Dresd. ber. 4, 44-54 (2008)

Brakman, S., Garretsen, H.: The relevance of initial conditions for German unification. Kyklos 46(2), 163-181 (1993)

Brakman, S., Garretsen, H.: Can Eastern Germany catch-up? A brief look at neo-classical and modern growth and trade theories. In: Blien, U., Herrmann, H., Koller, M. (Hrsg.) Regionalentwicklung und regionale Arbeitsmarktpolitik. Beiträge zur Arbeitsmarkt- und Berufsforschung, Bd. 184, S. 64-73. Nürnberg (1994)

Cliff, A., Ord, J.K.: Spatial Autocorrelation. Pion, London (1973)

Dauth, W.: The Mysteries of the Trade: Interindustry Spillovers in Cities. Analysing the Causes of MAR-Externalities using Spatial Econometric Techniques. Paper presented at the Spatial Econometrics Association Conference in Chicago (2010)

Duranton, G., Puga, D.: Micro-foundations of urban agglomeration economies. In: Henderson, J.V., Thisse, J.-F. (Hrsg.) Handbook of Regional and Urban Economics, Bd. 4, S 2063-2117. NorthHolland, Amsterdam (2004)

Elhorst, J.P.: The mystery of regional unemployment differentials: theoretical and empirical explanations. J. Econ. Surv. 17(5), 709-748 (2003)

Ellison, G., Glaeser, E.: Geographic concentration in US manufacturing industries: a dartboard approach. J. Polit. Econ. 105, 889-927 (1997)

Florax, R.J.G.M., Nijkamp, P.: Misspecification in Linear Spatial Regression Models. Tinbergen Institute Discussion Paper (2003)

Friedman, M.: Do old fallacies ever die? J. Econ. Lit. 30(4), 2129-32 (1992)

Glaeser, E.L., Kallal, H.D., Scheinkman, J.A., Shleifer, A.: Growth in cities. J. Polit. Econ. 100(6), 1125-1152 (1992)

Görmar, W., Irmen, E.: Nichtadministrative Gebietsgliederungen und -kategorien für die Regionalstatistik. Die siedlungsstrukturelle Gebietstypisierung der BfLR. Raumforsch. Raumordn. 49(6), 387-394 (1991)
Heiland, F.: Trends in east-west German migration from 1989 to 2002. Demogr. Res. 11, 174-193 (2004)

Henderson, V., Kuncoro, A., Turner, M.: Industrial development in cities. J. Polit. Econ. 103(5), 1067-1090 (1995)

Krugman, P.: Geography and Trade. MIT Press, Cambridge (Mass.) (1991)

Ludsteck, J.: VALA - das ökonometrische Modell. Soz. Fortschr. 55(11-12), 272-275 (2006)

Quah, D.T.: Galton's fallacy and tests of the convergence hypothesis. Scand. J. Econ. 95(4), 427-443 (1993)

Röhl, K.-H.: Strukturelle Konvergenz der ostdeutschen Wirtschaft. IWTrends 1 (2009)

Südekum, J.: Increasing returns and spatial unemployment disparities. Pap. Reg. Sci. 84(2), 159-181 (2005)

Uhlig, H.: Regional Labor Markets, Network Externalities and Migration: the Case of German Reunification. SFB 649 Discussion Paper 2006-004 (2006a)

Uhlig, H.: Regional labor markets, network externalities and migration: the case of German reunification. Am. Econ. Rev. 96(2), 383-387 (2006b)

Zenou, Y.: Urban Labour Economics. Cambridge University Press, Cambridge (2009)

Uwe Blien beendete 1980 das Studium der Volkswirtschaftslehre und Soziologie an der Universität Regensburg mit dem Diplom. 1986 promovierte er dort zum Doktor der Wirtschaftswissenschaft. Er war wissenschaftlicher Mitarbeiter an den Universitäten ErlangenNürnberg und Regensburg sowie am Zentrum für Umfragen, Methoden und Analysen (ZUMA) in Mannheim. 1999 habilitierte er für Volkswirtschaftslehre an der TU Kaiserslautern. 1990 begann er seine Tätigkeit für das Institut für Arbeitsmarkt- und Berufsforschung (IAB) in Nürnberg. Seit 2002 ist er Leiter des Forschungsbereichs Regionale Arbeitsmärkte. Im Jahr 2006 wurde er zum Honorarprofessor der TU Kaiserslautern berufen und seit 2008 ist er Professor an der Otto-Friedrich-Universität Bamberg. Uwe Blien ist Fellow des Labor and Socio-Economic Research Center (LASER) der Universität Erlangen-Nürnberg und Research Fellow des Forschungsinstituts zur Zukunft der Arbeit. Im Februar 2010 wurde er zum Vorsitzenden der „Gesellschaft für Regionalforschung“ (GfR) gewählt, dies ist die deutschsprachige Sektion der European Regional Science Association (ERSA).

E-Mail: uwe.blien@iab.de

Phan thi Hong Van studierte Ingenieurökonomie an der Universität in Hanoi (Abschluss 1986 als Diplomingenieurin). In den Jahren 1987-1995 war sie wissenschaftliche Mitarbeiterin im Institut für Arbeitswissenschaft und soziale Probleme des Arbeitsministeriums in Hanoi. Von 1996-2000 war sie als Gastwissenschaftlerin im IAB und an der TU Kaiserslautern. Seit Juli 2002 ist sie als Mitarbeiterin im IAB beschäftigt.

E-Mail: van.phan-thi-hong@iab.de

Klara Kaufmann schloss ihr Studium der Ökotrophologie in Fulda 1992 mit dem Diplom ab. Nach einer Tätigkeit im Naturkostbereich erwarb sie einen Abschluss in der System- und Datenbankadministration. Seit 2004 ist sie im IAB als Mitarbeiterin mit dem Schwerpunkt Analyse regionaler Arbeitsmärkte tätig.

E-Mail: klara.kaufmann@iab.de

Steffen Kaimer studierte Sozialwissenschaften mit dem Schwerpunkt empirische Sozialforschung. Ab 2000 war er freiberuflicher Berater für empirische Sozialforschung und Datenmanagement (u. a. für das ABIS-Projekt des IAB). Seit 2001 ist er wissenschaftlicher Mitarbeiter im Geschäftsbereich IT- und Informationsmanagement.

E-Mail: steffen.kaimer@iab.de 